\title{
Evaluation of Euler Fluxes for Hypersonic Heating Computations
}

\author{
Keiichi Kitamura* and Eiji Shima \\ Japan Aerospace Exploration Agency, Kanagawa 229-8510, Japan \\ Yoshiaki Nakamura \\ Nagoya University, Aichi 464-8603, Japan \\ and \\ Philip L. Roe $\underline{\S}$ \\ University of Michigan, Ann Arbor, Michigan 48109-2140 \\ DOI: $10.2514 / 1.41605$
}

\begin{abstract}
In hypersonic flow computations, it is a key issue to predict surface heating accurately, though this is still challenging because there always are possibilities of resulting in anomalous solutions. In this paper, three properties for flux functions are proposed: 1) shock stability/robustness, 2) conservation of total enthalpy, and 3) resolving boundary layer. Then, numerical experiments are performed for widely used or recently developed flux functions, and these fluxes are categorized into five major groups based on how they satisfy the three properties. These tests reveal that no flux function investigated here possesses all the three properties. In particular, the first one is not satisfied by any flux functions, including flux-vector-splittings. Finally, contributions of those properties are compared in a two-dimensional, viscous, hypersonic blunt-body problem. Results showed that the first and the third properties are crucial, and the second one is preferred to predict hypersonic heating. A group of flux functions that best satisfies these properties is suggested, and they are recommended either to be used or designed for hypersonic heating computations.
\end{abstract}

\section{Nomenclature}

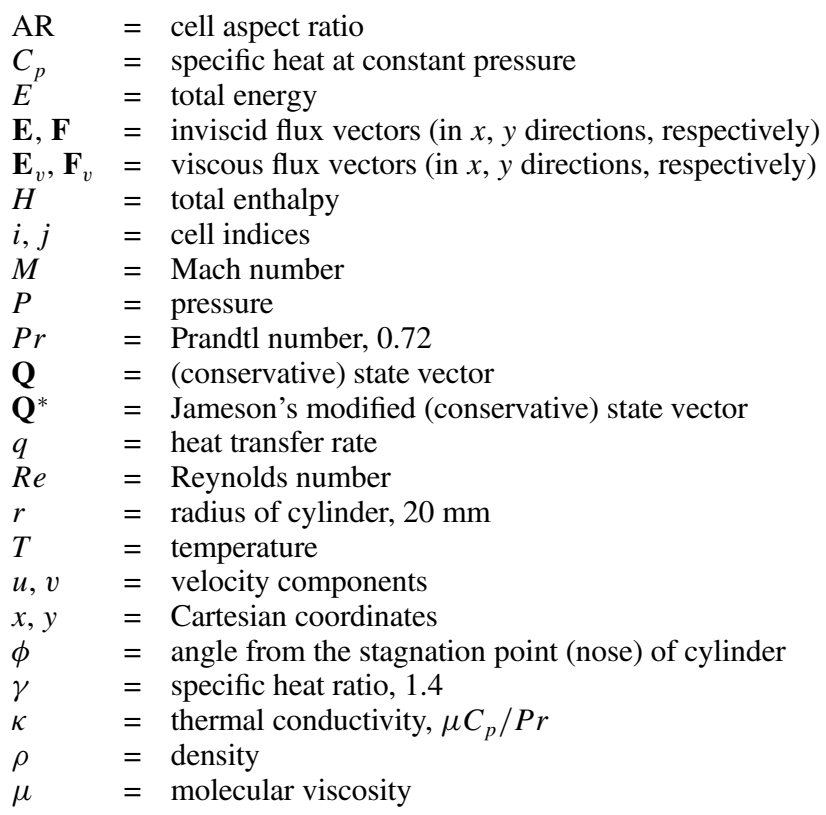

Received 14 October 2008; revision received 19 August 2009; accepted for publication 3 December 2009. Copyright (c) 2009 by the authors. Published by the American Institute of Aeronautics and Astronautics, Inc., with permission. Copies of this paper may be made for personal or internal use, on condition that the copier pay the $\$ 10.00$ per-copy fee to the Copyright Clearance Center, Inc., 222 Rosewood Drive, Danvers, MA 01923; include the code 0001-1452/10 and $\$ 10.00$ in correspondence with the CCC.

*Researcher, Engineering Digital Innovation Center, 3-1-1 Yoshinodai, Sagamihara; kitamura.keiichi@jaxa.jp. Member AIAA.

${ }^{\dagger}$ Senior Researcher and Director, Head of Team for Utilization of Simulation Technology, Engineering Digital Innovation Center, 3-1-1 Yoshinodai, Sagamihara. Senior Member AIAA.

Frofessor, Department of Aerospace Engineering, 1 Furo-cho, Chikusaku, Nagoya. Member AIAA.

${ }^{\S}$ Professor, Department of Aerospace Engineering. Fellow AIAA.

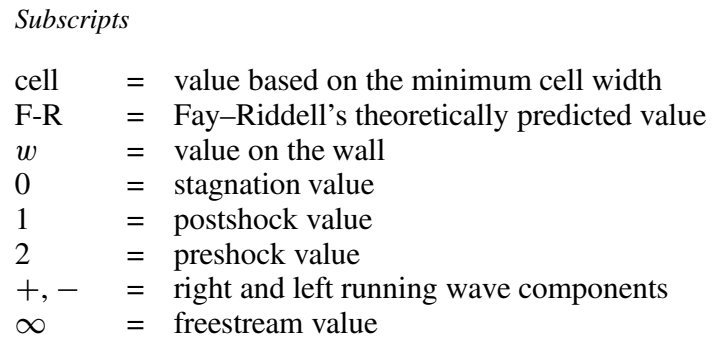

\section{Introduction}

T HYPERSONIC flow computations, it is a key issue to predict surface heating accurately. However, it is still challenging to compute hypersonic flows, because there are always possibilities of

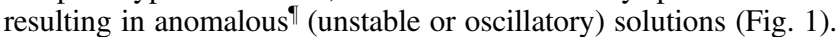
The most notorious example is the carbuncle phenomenon first reported by Peery and Imlay [1]. The carbuncle is a shock-induced instability and such anomalies appear depending on the following factors and their combinations [2,3]: flow conditions (Mach number, Reynolds number, and specific heat ratio), mesh (size, aspect ratio, etc.), and numerical methods (flux function, accuracy, etc.). In particular, the authors [4] recently made clear that there are at least two causes of the shock anomalies: one is one-dimensional (1-D), and the other is a multidimensional (MD) effect. The former appeared to be alleviated by satisfying the entropy condition (the second law of thermodynamics), whereas the latter can be suppressed by MD dissipation terms such as those introduced in [프, 6$]$. However, when both of the two causes arise at the same time, these dissipations do not work well. Thus, a flux function that is free from those two kinds of anomalies is needed, yet we have not had it. Any flux functions investigated in [4] can lead to at least either of onedimensional or MD anomalous solutions depending on the shock location relative to grid lines. Furthermore, Henderson and Menart [7] confirmed the report from Pandolfi and D'Ambrosio [2] that cell

\footnotetext{
"This expression has been replaced from unstable to stand for both unstable and oscillatory (private communication with Meng-Sing Liou, NASA John H. Glenn Research Center at Lewis Field, Jan. 2009). This will be used throughout the paper.
} 
aspect ratio (AR) plays a role in the occurrence of MD shock anomalies. They showed in Quirk's odd-even decoupling test [8] that elongation of cells in a direction parallel to the shock, and similarly, clustering the grids in a direction normal to the shock, are both an effective cure for the MD anomalies. However, investigations in both [2,7] are limited to flux functions known to be vulnerable to the shock anomalies; thus, an extension of their discussions to other fluxes is questionable. Therefore, in the present work, we will first extend our previous investigations [4] to a wider range of grids with different ARs and number of cells along with additional fluxes not tested in [4].

Moreover, Gnoffo and White [9] examined how asymmetry of surface heating appeared in tetrahedral unstructured grids. Mazaheri and Kleb [10] performed a grid study on hypersonic heating using initially shock-aligned but intentionally deformed structured grids. These studies showed that, even with their version of Roe flux [11], stagnation heating deviated as much as $18 \%$ associated with perturbation of grids and hence, captured shock shapes as well as boundary layers. As reviewed above, accurate computation of surface heat transfer rates is still one of extremely difficult subjects in computational fluid dynamics (CFD). This is partly due to the shock anomalies, but there remain other two factors to be considered: total enthalpy and boundary layers. If the total temperature numerically changes, and even if this error itself is insignificant, it could lead to poor prediction of the surface heating because the heat flux is proportional to temperature gradient from Fourier's law of heat conduction. Therefore, from this point of view, it would be preferred to adopt a flux function that is designed to conserve the total enthalpy across the shock wave, such as AUSM+ [12], AUSMPW+ [ㅁ] , RoeM [6], Hänel [13], and H-CUSP [14].

To capture the temperature gradient near the wall, we also should take into account the capability of resolving boundary layers. Fluxvector-splitting (FVS) fluxes (e.g., Stegar-Warming [15], van Leer Leer [16], and Hänel [13]) are known to lack this character because they are not formulated to incorporate effects of contact discontinuities. These fluxes are to be examined here.

Therefore, it is hypothesized that a flux function equipped with the following properties is suitable in hypersonic surface heat transfer computations: 1) shock stability/robustness (i.e., free from both onedimensional and MD anomalies [4,17]), 2) conservation of total enthalpy (and hence, total temperature, and 3) resolving boundary layer (and hence, temperature gradient near the wall).

In this paper, based on the three properties proposed above, we begin with investigating widely used or recently developed flux functions by conducting numerical experiments:

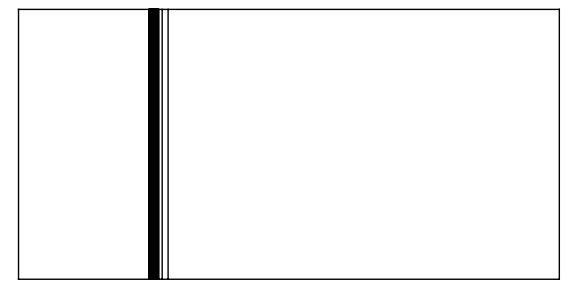

a)

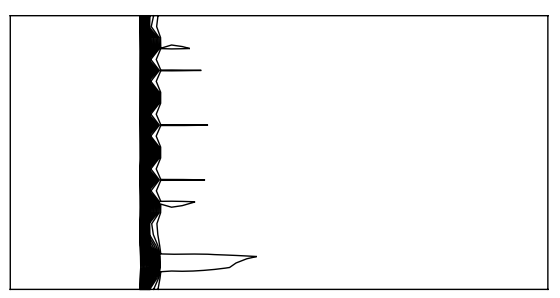

c)
1) The aforementioned extension of [4] will be conducted with regard to shock stability/robustness.

2) These fluxes will be tested for the constancy of total enthalpy in both one-dimensional and two-dimensional contexts with first and second order of spatial accuracy.

3) Laminar boundary layer over a flat plate will be solved and the solution accuracy will be compared. Then, flux functions are categorized into five groups depending on how they meet the three properties.

4) Contributions of those properties are compared in a twodimensional, viscous, hypersonic blunt-body problem.

Although individual significance of each of these properties has already been addressed by some researchers [2, $2,12-14]$, its direct influence on the resultant surface heating or interactions of each of the properties has not been examined yet. In other words, criteria for choosing fluxes are still unclear for hypersonic heating computations, despite the fact that a great number of flux functions have been developed. To establish these criteria, the previous work by the authors [4] has been extended here with the following features:

1) Three properties are introduced for heating computations, and Euler fluxes are evaluated comprehensively based on how they satisfy these properties.

2) In [4], only the first property was studied for limited cases. However, the current work covers a wider range of fluxes and grids, along with new findings and an improved rating for fluxes.

Therefore, provided here are very useful pieces of information for those who are currently using upwind schemes in their finite volume codes, as well as those who are developing CFD algorithms.

\section{Computational Method}

\section{A. Governing Equations}

The governing equations are the two-dimensional, compressible Euler or Navier-Stokes equations:

Euler:

$$
\frac{\partial \mathbf{Q}}{\partial t}+\frac{\partial \mathbf{E}}{\partial x}+\frac{\partial \mathbf{F}}{\partial y}=0
$$

Navier-Stokes:

$$
\frac{\partial \mathbf{Q}}{\partial t}+\frac{\partial \mathbf{E}}{\partial x}+\frac{\partial \mathbf{F}}{\partial y}=\frac{\partial \mathbf{E}_{v}}{\partial x}+\frac{\partial \mathbf{F}_{v}}{\partial y}
$$

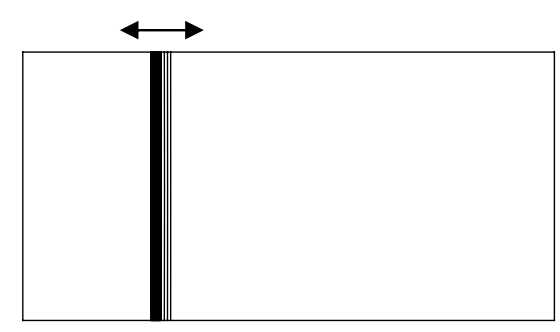

b)

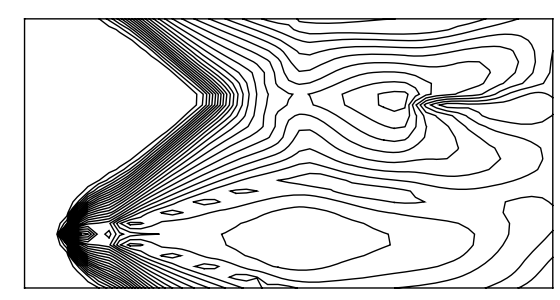

d)

Fig. 1 Plots of a) stable (2: symmetry and converged), b) one-dimensional anomaly (1: oscillatory), c) MD anomaly (1: asymmetry), and d) carbuncle (0: breakdown of shock) results for 1.5-dimensional steady shock test (first-order both in space and time; freestream Mach number $M_{\infty}=6.0$ ) [ $\left.\underline{4}\right]$. 


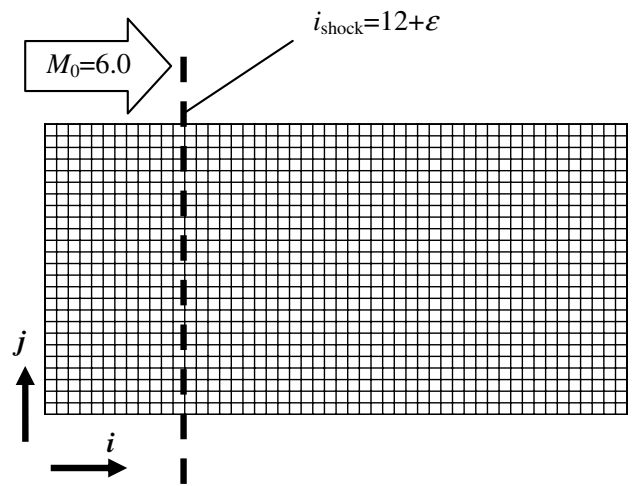

a)

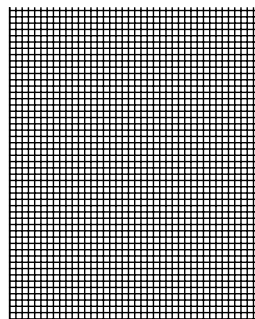

c)
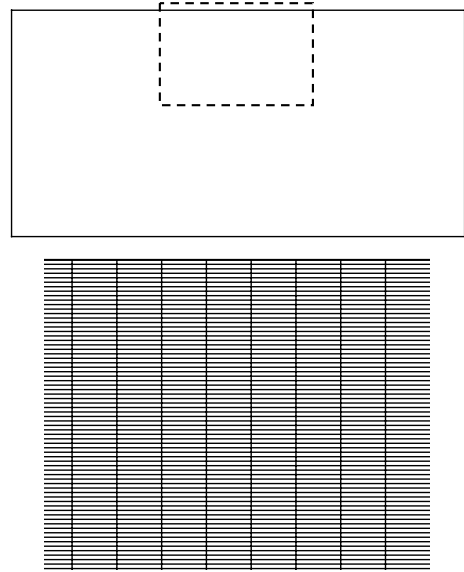

b)

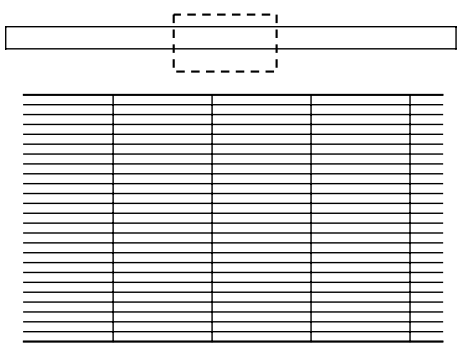

d)

Fig. 2 Illustrations of a) original $(50 \times 25)$, b) modified $1(50 \times 250$; $A R=10)$, c) modified $2(50 \times 250)$, and d $)$ modified $3(50 \times 25 ; A R=10)$ grids for 1.5-dimensional steady shock tests.

$$
\begin{gathered}
\mathbf{Q}=\left[\begin{array}{c}
\rho \\
\rho u \\
\rho v \\
\rho E
\end{array}\right], \quad \mathbf{E}=\left[\begin{array}{c}
\rho u \\
\rho u^{2}+p \\
\rho u v \\
\rho u H
\end{array}\right], \quad \mathbf{F}=\left[\begin{array}{c}
\rho v \\
\rho v u \\
\rho v^{2}+p \\
\rho v H
\end{array}\right] \\
\mathbf{E}_{v}=\left[\begin{array}{c}
0 \\
\tau_{11} \\
\tau_{21} \\
u_{j} \tau_{j k}+\kappa \frac{\partial T}{\partial x}
\end{array}\right], \quad \mathbf{F}_{v}=\left[\begin{array}{c}
0 \\
\tau_{12} \\
\tau_{22} \\
u_{j} \tau_{j k}+\kappa \frac{\partial T}{\partial y}
\end{array}\right] \\
\tau_{j k}=\mu\left[\left(\frac{\partial u_{j}}{\partial x_{k}}+\frac{\partial u_{k}}{\partial x_{j}}\right)-\frac{2}{3} \frac{\partial u_{l}}{\partial x_{l}} \delta_{j k}\right]
\end{gathered}
$$

where $\rho$ is density, $u$ and $v$ are velocity components in Cartesian coordinates, $E$ is total energy, $p$ is pressure, $H$ is total enthalpy $[H=E+(p / \rho)]$, and $T$ is temperature. The working gas is assumed to be air approximated by the calorically perfect gas model with the specific heat ratio $\gamma=1.4$. The Prandtl number is $\operatorname{Pr}=0.72$. The molecular viscosity $\mu$ is calculated by the Sutherland's formula, and the thermal conductivity $\kappa$ is given by $\kappa=\mu C_{p} / \operatorname{Pr}$, where $C_{p}$ is specific heat at constant pressure.

\section{B. Computational Method}

The following methods are used for computations herein, if not mentioned otherwise. As for spatial discretization, the primitive variables at each cell interface are interpolated to achieve secondorder accuracy by using MUSCL reconstruction [18] with van Albada et al.'s limiter [19]. Then inviscid fluxes at the cell interface are calculated from the following flux functions:

Group 1 (exact or three-wave approximate Riemann fluxes): Godunov, Roe, Roe (e-fix), and EC-Roe. Property 2 is no and property 3 is yes: 1) Godunov [20] is an exact Riemann solver,
2) Roe [11] is a three-wave approximate Riemann solver and a FDS scheme, 3) Roe (e-fix) is Roe with Harten's entropy fix [21], 4) ECRoe $(\alpha=0.2)[22-25]$ is an entropy-consistent Roe flux with a small amount of dissipation addition, and 5) EC-Roe $(\alpha=0.8)$ [22-25] is an entropy-consistent Roe flux with a large amount of dissipation addition.

Group 2 (two-wave approximate Riemann fluxes): Harten-Laxvan_Leer-Einfeldt (HLLE) and van Leer. Property 2 is no and property 3 is no. 1) HLLE [26] is a two-wave approximate Riemann solver, and a contact discontinuity is ignored, and 2) van Leer [16] is an FVS scheme, and a contact discontinuity is ignored.

Group 3 (total enthalpy-preserving, two-wave approximate Riemann flux): Hänel. Property 2 is yes and property 3 is no: 1) Hänel [13] is a variant of van Leer's flux, which preserves total enthalpy in steady flow.

Group 4 (total enthalpy-preserving fluxes): AUSMDV, AUSM $^{+}$, $\mathrm{AUSM}^{+}$-up, AUSMPW+, and RoeM. Property 2 is yes and property 3 is yes: 1) AUSMDV [27] is a variant of AUSM [28] (a simplified van Leer's FVS), but developed to have the boundarylayer-resolving nature of FDS and robustness of FVS, and to preserve total enthalpy $H$ in steady flow, 2) AUSM+ [12] is a variant of AUSM that preserves total enthalpy $H$ in steady flow and can also be regarded as a mixture of FDS and FVS, 3) $\mathrm{AUSM}^{+}$-up [29] is variant of AUSM + that is extended for use in low-speed flows, 4) AUSMPW + [5] is a variant of AUSM+ that features a multidimensional dissipation term, and 5) RoeM [6] is a variant of Roe that preserves total enthalpy $H$ and features a multidimensional dissipation term.

Group 5 (hybrid fluxes): AUSMDV (shock-fix) and RotatedRHLL. 1) AUSMDV (shock-fix) [27] is a combination of AUSMDV in the shock-normal direction and Hänel in the shock-parallel direction. These two fluxes should be manually selected by users. 2) Rotated-RHLL [30] is a hybrid of HLLE in the shock-normal 
Table 1 Summary of computed results for one-dimensional and 1.5-D $M_{\infty}=6.0$ steady shock tests with various flux functions

\begin{tabular}{|c|c|c|c|c|c|c|c|c|c|c|c|}
\hline \multirow[b]{2}{*}{ Test problem } & \multicolumn{10}{|c|}{$\varepsilon-$} & \multirow[b]{2}{*}{ Total } \\
\hline & 0.0 & 0.1 & 0.2 & 0.3 & 0.4 & 0.5 & 0.6 & 0.7 & 0.8 & 0.9 & \\
\hline \multicolumn{12}{|c|}{ Godunov (total 16) } \\
\hline 1-D & 1 & 2 & 2 & 2 & 2 & 2 & 2 & 1 & 1 & 1 & 16 \\
\hline $1.5-\mathrm{D}$ & 0 & 0 & 0 & 0 & 0 & 0 & 0 & 0 & 0 & 0 & 0 \\
\hline \multicolumn{12}{|c|}{ Roe (total 22) } \\
\hline $1-\mathrm{D}$ & 1 & 2 & 2 & 2 & 2 & 2 & 2 & 1 & 1 & 1 & 16 \\
\hline $1.5-\mathrm{D}$ & 0 & 2 & 2 & 2 & 0 & 0 & 0 & 0 & 0 & 0 & 6 \\
\hline \multicolumn{12}{|c|}{ Roe $(E-f i x)($ total 20) } \\
\hline $1-\mathrm{D}$ & 2 & 2 & 2 & 2 & 2 & 2 & 2 & 2 & 2 & 2 & 20 \\
\hline $1.5-\mathrm{D}$ & 0 & 0 & 0 & 0 & 0 & 0 & 0 & 0 & 0 & 0 & 0 \\
\hline \multicolumn{12}{|c|}{ EC-Roe $(\alpha=0.2)($ total 29$)$} \\
\hline 1-D & 2 & 2 & 2 & 2 & 2 & 1 & 1 & 1 & 1 & 2 & 16 \\
\hline $1.5-\mathrm{D}$ & 2 & 2 & 2 & 1 & 1 & 2 & 0 & 0 & 1 & 2 & 13 \\
\hline \multicolumn{12}{|c|}{ EC-Roe $(\alpha=0.8)($ total 20$)$} \\
\hline $1-\mathrm{D}$ & 2 & 2 & 2 & 2 & 2 & 2 & 2 & 2 & 2 & 2 & 20 \\
\hline $1.5-\mathrm{D}$ & 0 & 0 & 0 & 0 & 0 & 0 & 0 & 0 & 0 & 0 & 0 \\
\hline \multicolumn{12}{|c|}{ HLLE (total 32) } \\
\hline 1-D & 1 & 2 & 2 & 2 & 2 & 2 & 2 & 1 & 1 & 1 & 16 \\
\hline $1.5-\mathrm{D}$ & 1 & 2 & 2 & 2 & 2 & 2 & 2 & 1 & 1 & 1 & 16 \\
\hline \multicolumn{12}{|c|}{ van Leer (total 40) } \\
\hline 1-D & 2 & 2 & 2 & 2 & 2 & 2 & 2 & 2 & 2 & 2 & 20 \\
\hline $1.5-\mathrm{D}$ & 2 & 2 & 2 & 2 & 2 & 2 & 2 & 2 & 2 & 2 & 20 \\
\hline \multicolumn{12}{|c|}{ Hänel (total 40) } \\
\hline 1-D & 2 & 2 & 2 & 2 & 2 & 2 & 2 & 2 & 2 & 2 & 20 \\
\hline 1.5-D & 2 & 2 & 2 & 2 & 2 & 2 & 2 & 2 & 2 & 2 & 20 \\
\hline \multicolumn{12}{|c|}{$A U S M D V($ total 30$)$} \\
\hline 1-D & 2 & 2 & 2 & 2 & 2 & 2 & 2 & 2 & 2 & 2 & 20 \\
\hline $1.5-\mathrm{D}$ & 1 & 1 & 1 & 1 & 1 & 1 & 1 & 1 & 1 & 1 & 10 \\
\hline \multicolumn{12}{|c|}{$\mathrm{AUSM}^{+}($total 33) } \\
\hline 1-D & 2 & 2 & 2 & 2 & 1 & 1 & 1 & 2 & 2 & 2 & 17 \\
\hline 1.5-D & 2 & 2 & 2 & 2 & 1 & 1 & 1 & 1 & 2 & 2 & 16 \\
\hline \multicolumn{12}{|c|}{$\mathrm{AUSM}^{+}$-up (total 33) } \\
\hline 1-D & 2 & 2 & 2 & 2 & 2 & 1 & 1 & 1 & 2 & 2 & 17 \\
\hline 1.5-D & 2 & 2 & 2 & 2 & 2 & 1 & 1 & 1 & 1 & 2 & 16 \\
\hline \multicolumn{12}{|c|}{ AUSMPW + (total 35) } \\
\hline 1-D & 2 & 2 & 2 & 2 & 2 & 2 & 2 & 1 & 1 & 1 & 17 \\
\hline $1.5-\mathrm{D}$ & 2 & 2 & 2 & 2 & 2 & 2 & 2 & 1 & 1 & 2 & 18 \\
\hline \multicolumn{12}{|c|}{ RoeM (total 28) } \\
\hline $1-\mathrm{D}$ & 1 & 2 & 2 & 2 & 2 & 2 & 2 & 2 & 1 & 1 & 17 \\
\hline $1.5-\mathrm{D}$ & 1 & 1 & 1 & 1 & 1 & 2 & 1 & 1 & 1 & 1 & 11 \\
\hline \multicolumn{12}{|c|}{ AUSMDV (shock fix) (total 40) } \\
\hline 1-D & 2 & 2 & 2 & 2 & 2 & 2 & 2 & 2 & 2 & 2 & 20 \\
\hline $1.5-\mathrm{D}$ & 2 & 2 & 2 & 2 & 2 & 2 & 2 & 2 & 2 & 2 & 20 \\
\hline \multicolumn{12}{|c|}{ Rotated-RHLL (total 36) } \\
\hline 1-D & 1 & 2 & 2 & 2 & 2 & 2 & 2 & 1 & 1 & 1 & 16 \\
\hline $1.5-\mathrm{D}$ & 2 & 2 & 2 & 2 & 2 & 2 & 2 & 2 & 2 & 2 & 20 \\
\hline
\end{tabular}

direction and Roe (e-fix) in the shock-parallel direction. These two fluxes are automatically activated depending on the relative direction of the shock to the cell-interface.

These fluxes have been categorized into the above five groups based on how the second and the third properties are satisfied, and the flux is whether a single or hybridized one. Note that property 1 was not used for the grouping because this property cannot be answered yes or no, but we shall rate those fluxes by quantizing them in the next section.

Viscous fluxes are computed by using second-order central difference, while for time integration, second-order Runge-Kutta

Table 2 Summary of computed results for original and modified $1.5-D M_{\infty}=6.0$ steady shock tests with various flux functions

\begin{tabular}{lcccc}
\hline \hline Scheme & $\begin{array}{c}\text { Original } \\
(\varepsilon=0.0)\end{array}$ & $\begin{array}{c}\text { Modified 1 } \\
(\varepsilon=0.0)\end{array}$ & $\begin{array}{c}\text { Modified 2 } \\
(\varepsilon=0.0)\end{array}$ & $\begin{array}{c}\text { Modified 3 } \\
(\varepsilon=0.0)\end{array}$ \\
\hline HLLE & 1 & 1 & - & - \\
van Leer & 2 & 1 & - & - \\
Hänel & 2 & 1 & - & - \\
$\quad \begin{array}{l}\text { AUSMDV } \\
\quad \text { (shock fix) }\end{array}$ & 2 & 1 & - & - \\
Rotated-RHLL & 2 & 1 & - & - \\
\hline \hline
\end{tabular}

method, or lower-upper symmetric Gauss-Seidel (LU-SGS) [31] is employed.

\section{Three Properties for Hypersonic Surface Heating Computations}

\section{A. Property 1: Shock Stability/Robustness}

1. One-Dimensional and Multidimensional Shock Anomalies

As previously mentioned, there are at least two kinds of shock anomalies at work: that is, 1-D and MD modes. The 1.5-D test (Fig. 2 ) in [4], in which one-dimensional shock is located in a twodimensional uniform grid (consisting of squares without perturbation), was shown to be very effective in investigating those two anomalies separately (Fig. 1). It was also demonstrated therein that the 1.5-D test can roughly but successfully predict the outcome of a two-dimensional blunt-body simulation, in terms of shock anomalies. Thus, we start from reviewing the results of $[4,30]$, and then carry out additional cases. The freestream Mach number is $M_{\infty}=6.0$, and the computations are conducted for 40,000 steps with Courant-Friedrichs-Lewy number of 0.5 using first-order schemes both in space and time. Detailed explanation for the computational setup is found in [4]. 


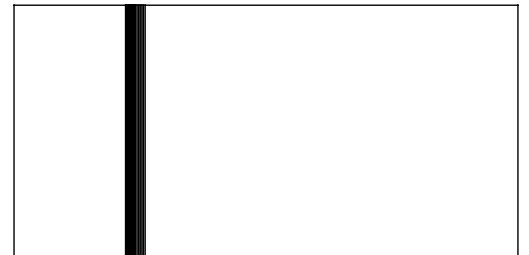

a)

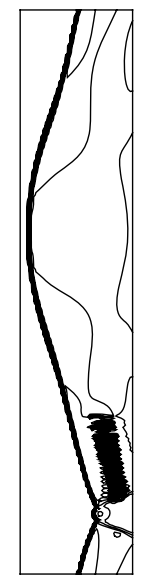

c)

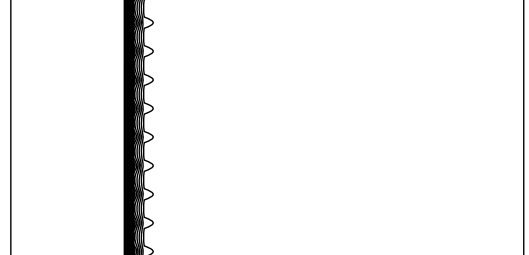

b)

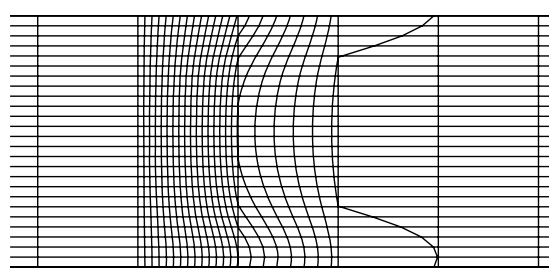

d)

Fig. 3 Plots of a) original (50 $\times 25)$, b) modified $1(50 \times 250$; AR $=10)$, c) modified $2(50 \times 250)$, and d) modified $3(50 \times 25$; AR = 10) 1.5-dimensional tests (van Leer's FVS, 200,000 steps [16]).

The results in $[4,30]$ are summarized as follows:

1) All the flux functions investigated there exhibited onedimensional oscillation, except for Roe (e-fix) and EC-Roe ( $\alpha=0.8$; $\alpha$ stands for amount of dissipation addition [22-25]). These exceptions were the ones formulated to satisfy the entropy condition across the shock.

2) One-dimensional oscillation was confined in one mesh size in a normal direction to the shock.

3) HLLE did not show MD anomalies, whereas it did in onedimensional mode (to be corrected later).

4) MD dissipation terms in AUSMPW+ or RoeM can partly suppress MD anomalies under certain conditions, but are not effective in one-dimensional anomalies.

5) These anomalies appear depending on the relative location of the shock with the grid lines.

6) Rotated RHLL, inherently multidimensional, did not show MD anomalies, at least in their tests (to be corrected later).

Then we conducted the same survey for other fluxes: FVS schemes of van Leer [16] and Hänel [13], and AUSMDV [27]. As is widely believed, FVS fluxes are considered to be free from either of the shock anomalies. AUSMDV, combined with Hänel (so-called shock fix), is also claimed as carbuncle-free.

7) The result is that FVS and AUSMDV (shock fix) fluxes did not show one-dimensional and MD anomalies (to be corrected later).

Both of the present and previous $[4,30]$ results are summarized in Table 1 . Note that the notations for the rating system have been improved from the previous form of S (stable), A (asymmetry), and U (unstable) [4] to the following: 2 represents stable and symmetric solutions with at least three orders of density residual reduction, 1 represents asymmetry or oscillation of the shock confined in two cells of the shock-normal direction, and 0 represents unstable solutions usually associated with total breakdown of the shock (carbuncle). The residual stagnated at a significant value.

These points will be used later in the comprehensive evaluation of the flux functions.

\section{Modified 1.5-D Tests}

Now we consider the more crude extension of the 1.5-D test, in which 10 times the cells in the shock-parallel direction are packed in the same domain; the cell aspect ratio (AR) is taken as 10 (Fig. 2b). Modification of this kind for grids can, as shown in [2,7], provoke MD shock anomalies. In the present test (referred to as modified test 1 , shock location parameter [4] is set to be $\varepsilon=0.0$, that is, the shock is initially put exactly on the grid line (cell interface). Then computations are conducted 200,000 time steps, which is five times as long as the original test, with $\mathrm{CFL}=0.5$.

The results are summarized in Table 2 , and because there was no major difference found from the result of one flux to another, only the van Leer's case is shown in Fig. 3b, along with the results of the original test for comparison (Fig. 3a). Surprisingly, even FVS fluxes and AUSMDV (shock fix) that were believed to be carbuncle-free showed MD oscillations in this modified setup. This tendency is consistent with Pandolfi and D'Ambrosio's finding [2], but their discussion was limited for a flux that was already known to suffer from carbuncles. The results also showed that all the fluxes that passed the (original) 1.5-D test failed in the modified 1.5-D test. RHLL, which showed no unacceptable results in the original test, yielded slight asymmetry (not shown due to space limitation). Furthermore, HLLE flux, which showed only a one-dimensional

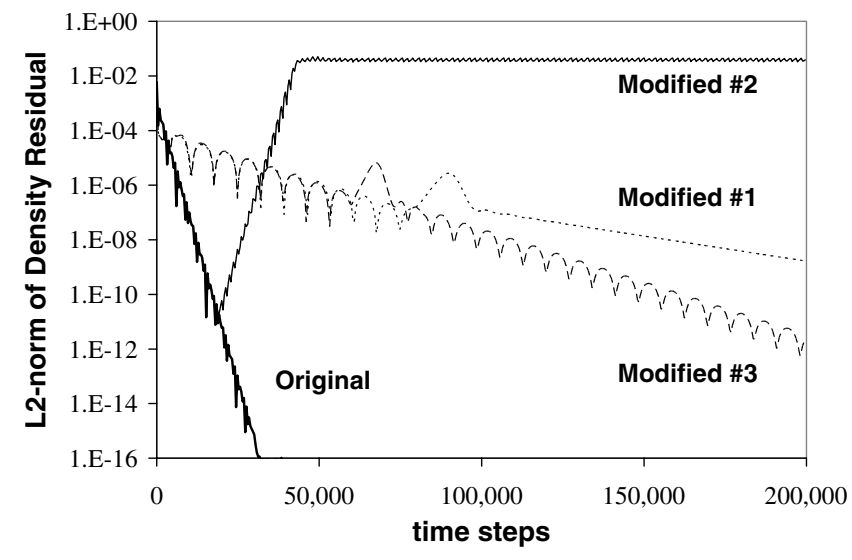

Fig. 4 Residual histories for original and modified 1.5-dimensional tests (van Leer [16]). 


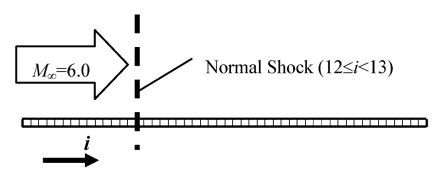

a)

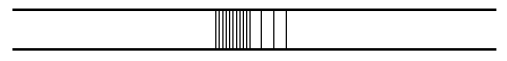

b)

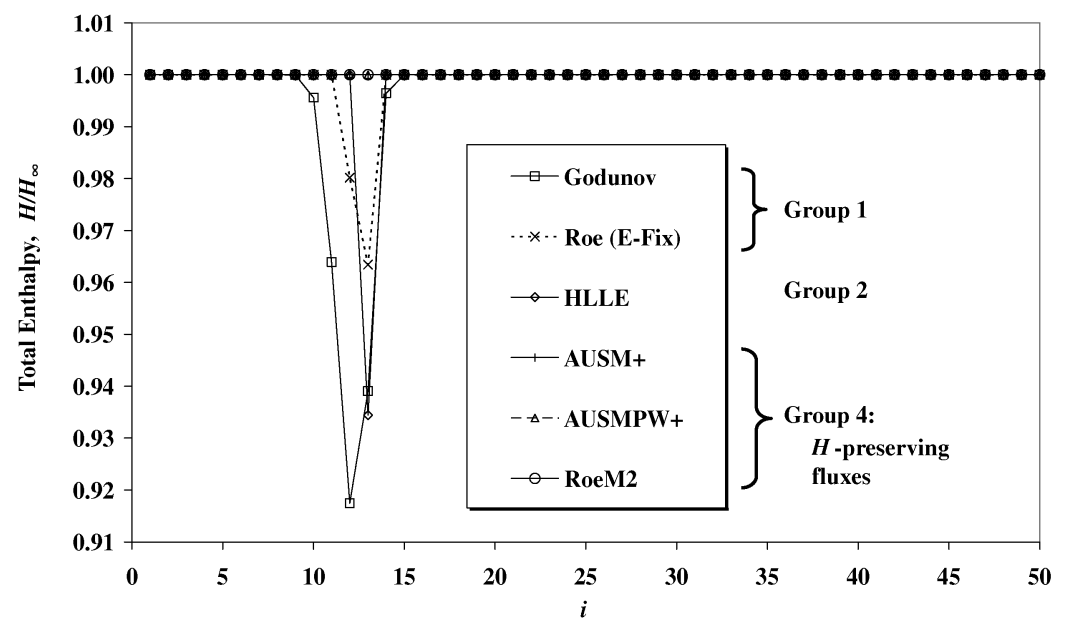

c)

Fig. 5 Total enthalpy-preserving capabilities of flux functions across normal shock: a) grid system, b) typical Mach number contours [4] and c) computed total enthalpy profiles.
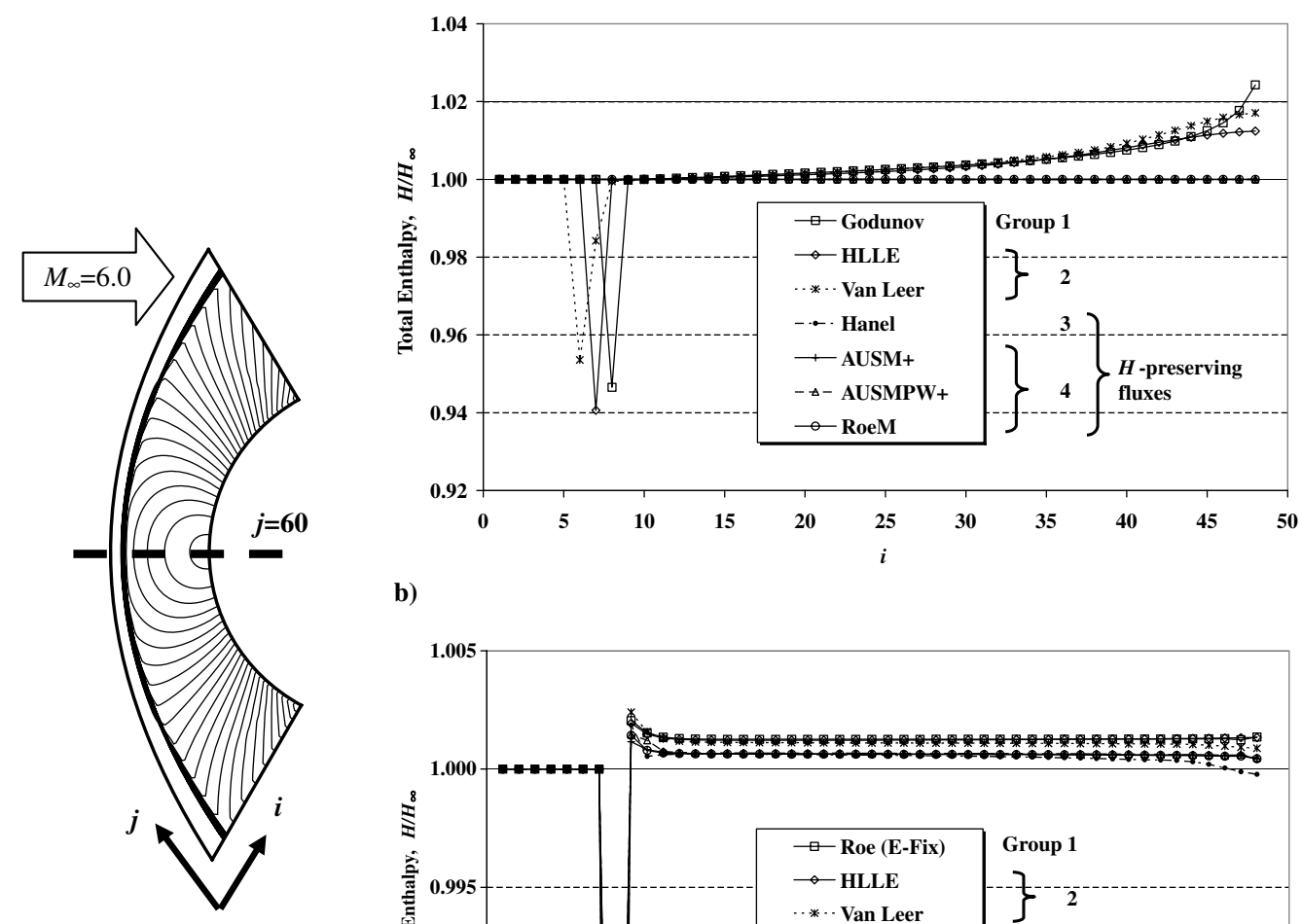

b)

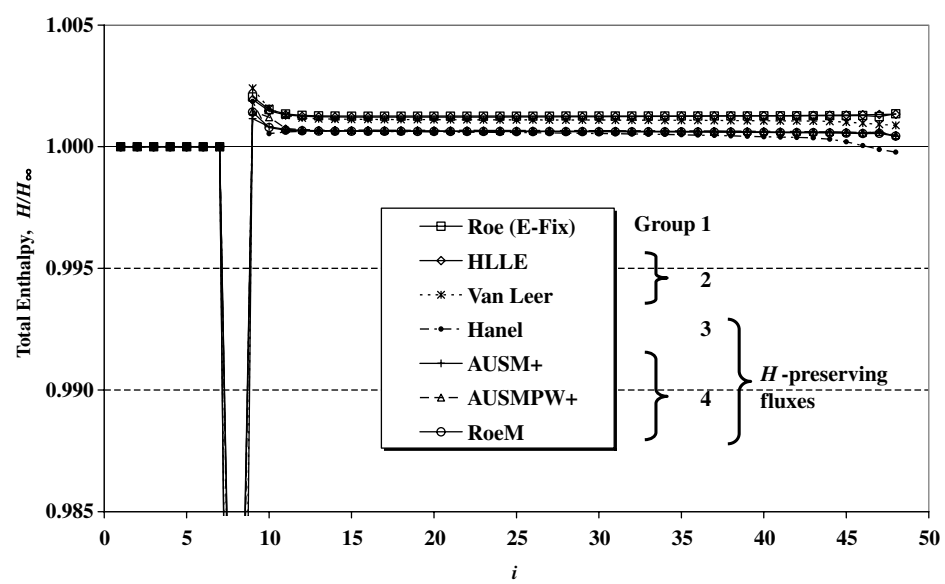

c)

Fig. 6 Total enthalpy-preserving capabilities of flux functions across bow shock: a) typical pressure contours []], and computed total enthalpy profiles $(j=60)$ of b) first-order and c) second-order results. 


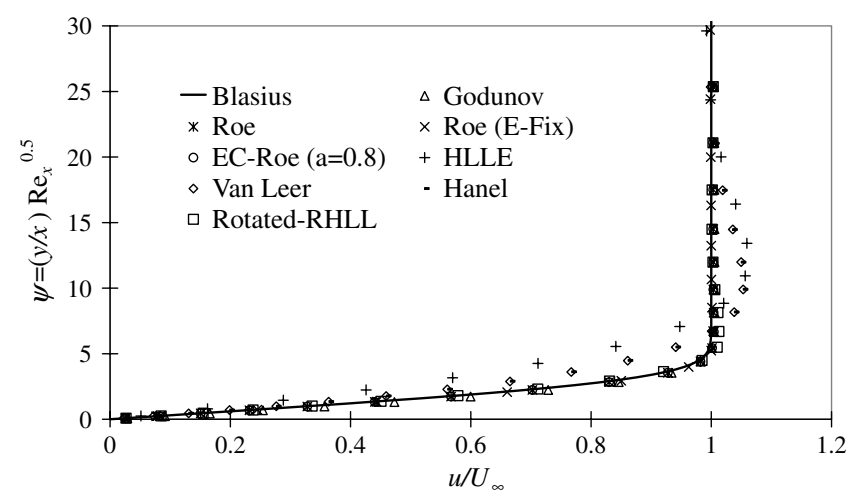

a)

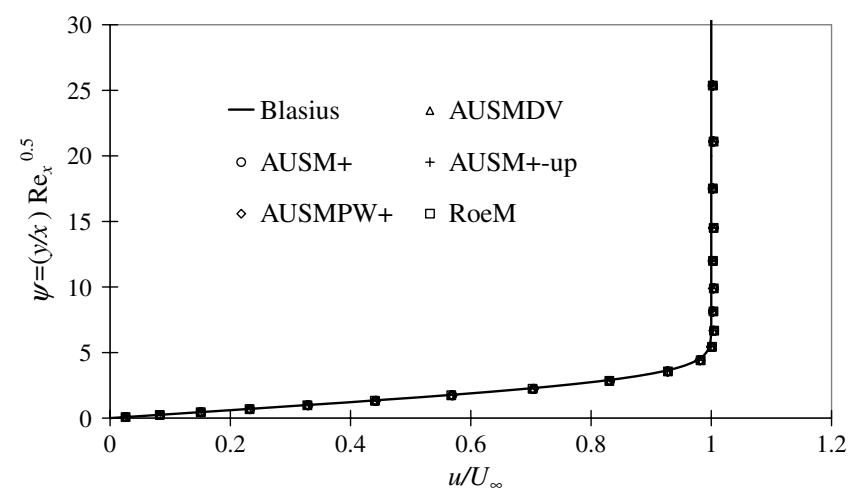

b)

Fig. 7 Boundary-layer resolution capabilities of flux functions.

mode of the shock oscillations in the original test, also exhibited MD mode in the present test (not shown again). It turned out that the items 3,6 , and 7 in the previous subsubsection were false. These items are corrected as follows: in item 3, HLLE rarely shows MD anomalies, and it sometimes doesone-dimensional mode. In item 6, rotated RHLL rarely shows MD anomalies. In item 7, FVS and AUSMDV (shock fix) fluxes rarely show MD anomalies, and they never do one-dimensional mode. Thus, we have no fluxes that are free from shock anomalies. Moreover, we also found similar results in the following:

1) Modified test 2: The computational domain is extended in the shock-parallel direction by 10 times in which the number of the cells is increased, but the AR is maintained (Fig. 2c).
2) Modified test 3: The computational domain is compressed to one-tenth the height in which the number of the cells are unmodified, and the AR is taken as 10 (Fig. 2d).

In particular, according to the results in Figs. $3 \mathrm{c}$ and $\underline{3 d}$, the former case appears to be more catastrophic. Therefore, findings in [2,7] are true, but the increment of the cell numbers in the shock-parallel direction actually plays a more significant role than the AR in the present cases. Even worse, the result tells that any flux functions have the potential to yield full carbuncle solutions, as in Fig. 3c.

In addition, the anomalous solutions emerged after apparently satisfactory solutions were once obtained. This is confirmed from residual (L2-norm of density) histories shown in Fig. 4, in which the residuals in the cases of the modified tests suddenly began to deviate from the original (stable) case. This is consistent with the behavior of relatively robust fluxes such as AUSM+ and RoeM in [4], in which the MD instability grew very gently.

Unless the grid lines are aligned well with the captured shock as in the most practical situations (and, of course, in unstructured meshes), it will be much harder to predict whether the computation will reach a stable or an unstable/oscillatory solution. For example, the more grid points are used near the shock, the more likely that the MD shock anomalies will be invoked in certain cases. This will be demonstrated later. Nevertheless, we will use only the results of the original test for the present evaluation of flux functions.

\section{B. Property 2: Conservation of Total Enthalpy}

As mentioned in the Introduction, if a flux function is not designed to preserve total enthalpy $H$, the stagnation temperature $T_{0}$, and hence the calculated wall heat transfer rates $q_{w}$, may include significant errors. This aspect of the flux functions was already claimed by other researchers $[6,14]$, but its importance has not yet been demonstrated in a quantitative sense. Thus, comparisons of various fluxes for this property are made here both in onedimensional and two-dimensional contexts.

As pointed out by Jameson [14], this property is satisfied, for instance, by modification of the state vector as follows:

$$
\mathbf{Q}=\left[\begin{array}{c}
\rho \\
\rho u \\
\rho v \\
\rho E
\end{array}\right] \rightarrow \mathbf{Q}^{*}=\left[\begin{array}{c}
\rho \\
\rho u \\
\rho v \\
\rho H
\end{array}\right]
$$

This modification is regarded as changing equations to be solved from the energy equation [consisting of the fourth row of Eq. (2)] to the equation of conservation of products of mass flux and total enthalpy: that is, the equation of total enthalpy conservation [because

Table 3 Evaluation of Euler fluxes based on three properties for hypersonic heating: groups 1-3

\begin{tabular}{|c|c|c|c|c|c|c|c|c|}
\hline \multirow[b]{2}{*}{ Flux functions } & \multicolumn{5}{|c|}{ Group 1} & \multicolumn{2}{|c|}{ Group 2} & \multirow{2}{*}{$\begin{array}{c}\text { Group } 3 \\
\text { Hänel }\end{array}$} \\
\hline & Godunov & Roe & EC-Roe $\alpha=0.2$ ) & Roe (E-fix) & EC-Roe $(\alpha=0.8)$ & HLLE & van Leer & \\
\hline \multicolumn{9}{|c|}{ I. Shock stability/robustness } \\
\hline 1-D (20) & 16 & 16 & 16 & 20 & 20 & 16 & 20 & 20 \\
\hline MD (20) & 0 & 6 & 13 & 0 & 0 & 16 & 20 & 20 \\
\hline II. $H$-preserving (10) & 0 & 0 & 0 & 0 & 0 & 0 & 0 & 10 \\
\hline III. B-L resolution (30) & 30 & 30 & 30 & 30 & 30 & 0 & 0 & 0 \\
\hline Grand total (80) & 46 & 52 & 59 & 50 & 50 & 32 & 40 & 50 \\
\hline
\end{tabular}

Table 4 Evaluation of Euler fluxes based on three properties for hypersonic heating: group 4

\begin{tabular}{lccccc}
\hline \hline & \multicolumn{5}{c}{ Group 4 } \\
\cline { 2 - 6 } Flux functions & AUSMDV & AUSM+ & AUSM+-up & AUSMPW+ & RoeM \\
\hline I. Shock stability/robustness & 20 & 17 & 17 & 17 & 17 \\
1-D (20) & 10 & 16 & 16 & 18 & 11 \\
MD (20) & 10 & 10 & 10 & 10 & 10 \\
II. H-preserving (10) & 30 & 30 & 30 & 30 & 30 \\
III. B-L resolution (30) & 70 & 73 & 73 & 75 & 68 \\
Grand total (80) & & & & \\
\hline \hline
\end{tabular}


the mass flux is already conserved in the first row of the Eq. (2)]. This strategy was adopted in H-CUSP [14] and also later in RoeM [6].

AUSM family schemes (AUSMDV [27], AUSM+ [12], $\mathrm{AUSM}^{+}$. up [29], AUSMPW ${ }^{+}$[5], etc.) and Hänel et al. [13] employed another approach. In their formulations, total enthalpy $\bar{H}$ is not differentiated, but directly used. The final component of the flux vector can be expressed as

$$
E_{4}=f^{+}(\rho u) \cdot H^{+}+f^{-}(\rho u) \cdot H^{-}
$$

We call these flux functions $H$-preserving fluxes (groups 3 and 4 ) in the rest of the paper, and this property is not accommodated in other popular fluxes (non- $H$-preserving fluxes, groups 1 and 2): for example, Godunov [20], Roe [11], and van Leer [16].

Figure $\underline{5}$ presents total enthalpy profiles across normal shock in one-dimensional setup. The computations shown here are that of [4], conducted with first-order schemes both in space and time. In [4] each flux function yielded either stable or oscillatory results depending on the shock position, but here $H$ profiles are extracted only from the stable cases. As demonstrated here, the $H$-preserving fluxes showed constancy of total enthalpy even inside the shock where the Euler equations are no longer valid [3,4,32], whereas the other fluxes exhibited oscillations. Nevertheless, those values recovered to their original states past the shock, showing that the $\mathrm{H}$ preserving property has minor effects in this test.

On the other hand, in the two-dimensional setup $(48 \times 120$ cells, Fig. $6 a)$, the total enthalpy calculated by a non- $H$-preserving flux deviated at most $3 \%$ downstream the shock as well as inside the shock along $j=60$ cells [slightly below the symmetry line that lies on the interfaces of $j=60$ and 61 cells (Fig. 6b)]. These deviations are, however, greatly suppressed when second-order reconstruction is employed (Fig. 6c: note that the scale differs from Fig. 6b). This is because these errors are of the order of truncation, as explained in

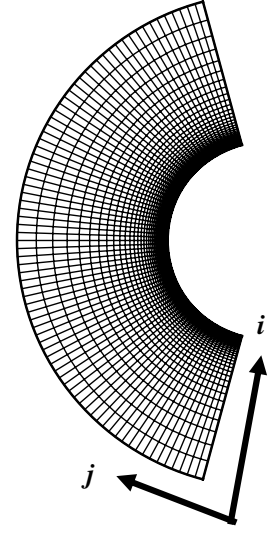

a)

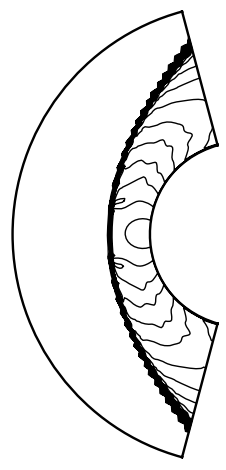

c)

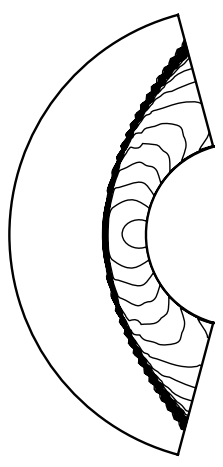

g)

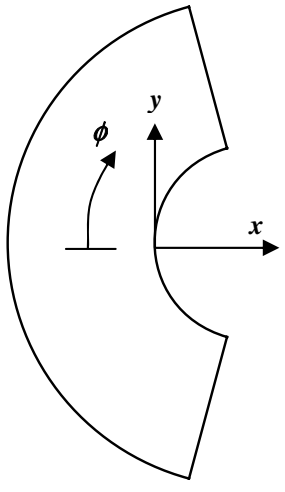

b)

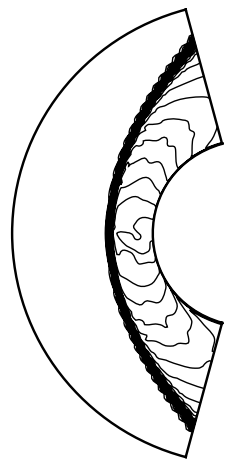

d)

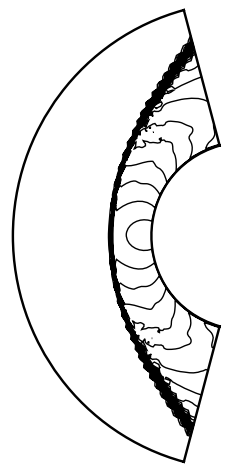

h)

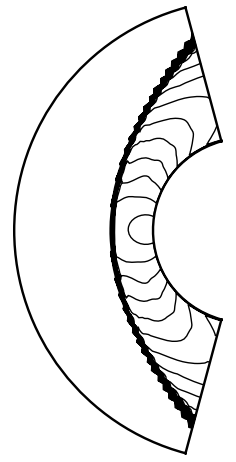

e)

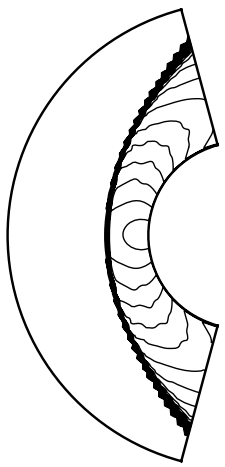

i)

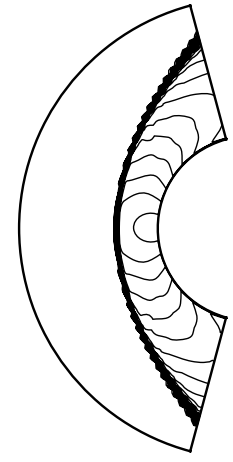

f)

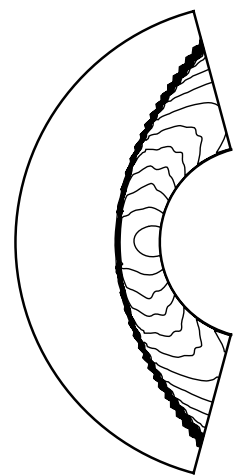

j)

Fig. 8 Illustrations of a) grid (every other grid lines are shown), b) coordinates, and results (Mach number contours at 100,000 steps, baseline grid) of c) Roe (e-fix), d) EC-Roe ( $\alpha=0.8)$, e) HLLE, f) van Leer [16], g) Hänel [13], h) AUSM+, i) AUSMPW+, and j) RoeM for blunt-body viscous test. 
[33], although these fluxes still produce larger errors than $H$ preserving schemes. In addition, it is noted that even $H$-preserving fluxes suffer from oscillation of total enthalpy inside the shock. Thus, we can say that the $H$-preserving nature does improve the total enthalpy profiles, and accordingly, surface heating, but that its influence is dramatically reduced with second-order (and presumably, higher-order) reconstruction. More practical comparisons for viscous cases will be performed later.

\section{Property 3: Boundary-Layer Resolution}

To resolve boundary layers is, of course, considered to be crucial for accurate prediction of surface heat transfer rates, because the heat flux is proportional to temperature gradient in the boundary layer. This property is not enjoyed by two-wave approximate Riemann fluxes (groups 2 and 3 ) due to ignorance of a contact surface in the formulation.

To confirm this property of each flux function, we conducted computations on a low-speed flow over a flat plate as in [30]. The flow conditions are $M_{\infty}=0.2, P_{\infty}=1.0128 \times 10^{5} \mathrm{~Pa}, T_{\infty}=$ $294.4 \mathrm{~K}$, and $R e_{x}=2.19 \times 10^{4}$ (Reynolds number based on where velocity profiles are extracted). The second-order-accurate, van Albada-limited MUSCL reconstruction $(\kappa=1 / 3)$ is adopted for cell-interface values, along with the second-order central difference for viscous term, and the second-order Runge-Kutta for temporal integration. The computations were conducted for 50,000 steps with $\mathrm{CFL}=0.5$, and all the computations achieved at least two-order reductions of the residuals (L2-norm of density). The results of different fluxes are compared in Fig. 7, as well as Blasius' analytical solution for a laminar boundary layer. As shown in these figures, most of the methods successfully reproduced the analytical velocity profile, and only two-wave solvers (groups 2 and 3) failed, as expected from their formulations.

All of the above results are summarized in Tables 3 and 4 along with overall ratings with the grand total of 80 points. As for property 1 , the sum of points obtained for each flux (Tables $\underline{3}$ and $\underline{4}$ ) is

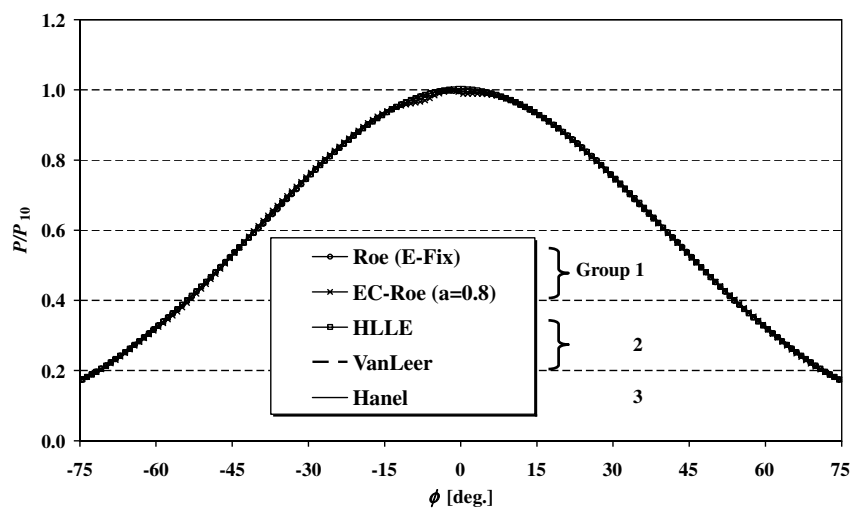

a)

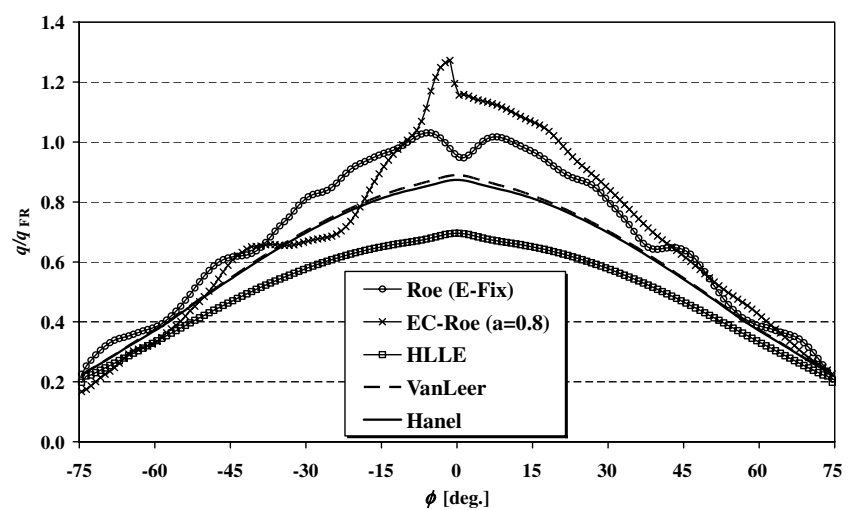

c)

Fig. 9 Profiles of a-b) pressure and c-d) heat transfer rates over blunt body (100,000 steps, baseline grid). satisfying all the three properties. diction of surface heat transfer rates using these fluxes.

b)

d)

employed. The scaling of rating for each property is proposed based on its impact on the surface heating (explained later). As can be seen, no flux functions investigated here are found to be completely

The classification of fluxes discussed above is also included in Tables $\underline{3}$ and $\underline{4}$. This table provides very useful information to those who are using and designing upwind schemes. Having addressed three properties of flux functions and categorized 15 fluxes into five groups, we will show hypersonic viscous cases with regard to pre-

Considering the fact that any combinations are generally possible for hybrid methods, we will focus on only single fluxes that can be candidate components of hybrid methods in the rest of the paper.

\section{Hypersonic Heating Test: Hypersonic Viscous Flow over a Blunt Body}

In this section, two-dimensional, viscous, hypersonic flow computations over a blunt body (a circular cylinder) are conducted. To explain suggested weighting of the three properties in Tables 3 and 4 , comparisons are made for fluxes from different groups from 1 to 4 . The surface heat transfer rates computed by different fluxes from each group are also compared with theory. In contrast to [4], we used a grid not aligned with a theoretical shock shape, because shock alignment is practically impossible, for instance, in shock-shock interacting problems [34-38]. For the shock-aligned grid cases (see [39]).

We consider the experimental setup of a two-dimensional blunt body with $r=20 \mathrm{~mm}$ radius mounted in Nagoya University Shock Tunnel [40], in which the freestream conditions are $M_{\infty}=8.1$, $P_{\infty}=370.7 \mathrm{~Pa}, T_{\infty}=63.73 \mathrm{~K}$, and $R e=1.3 \times 10^{5}$ (Reynolds number based on the radius $r=20 \mathrm{~mm}$ ). The present computation employed the exact same freestream conditions. In addition, no-slip and isothermal $\left(T_{w}=300 \mathrm{~K}\right)$ conditions are imposed at the wall. The cell Reynolds number, which is based on the minimum cell size,
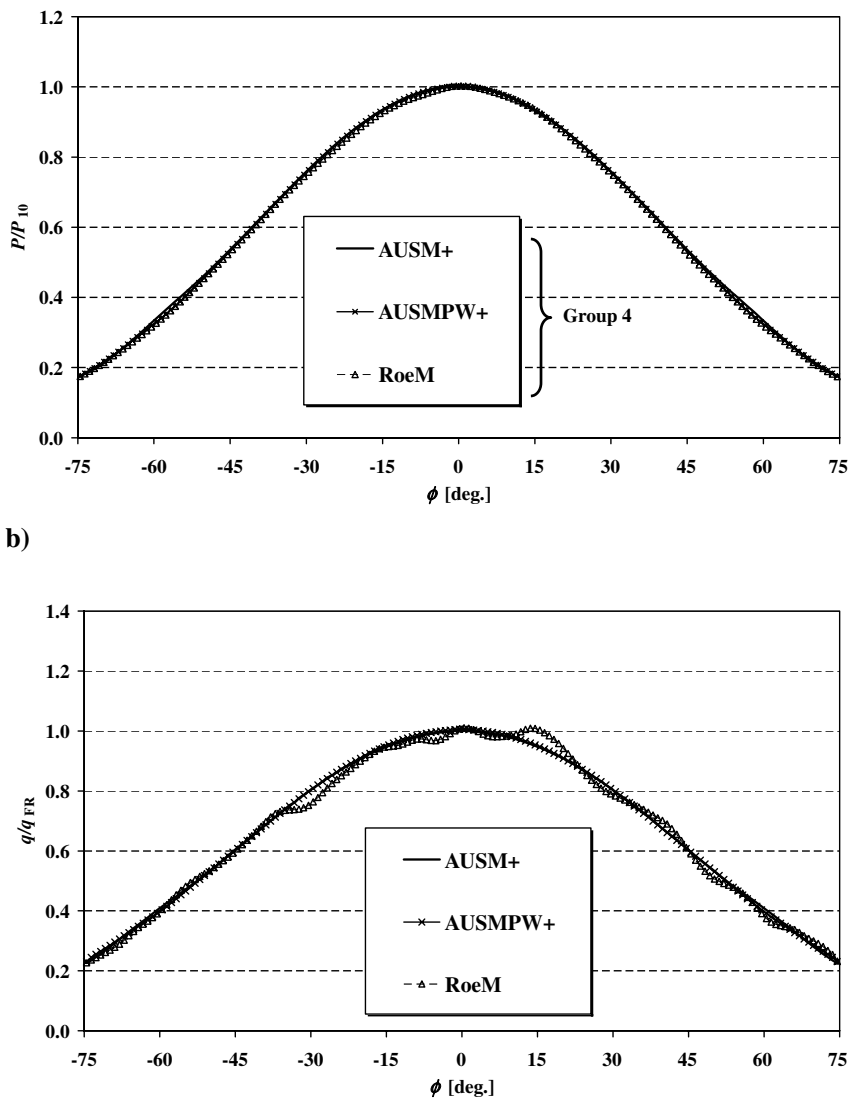


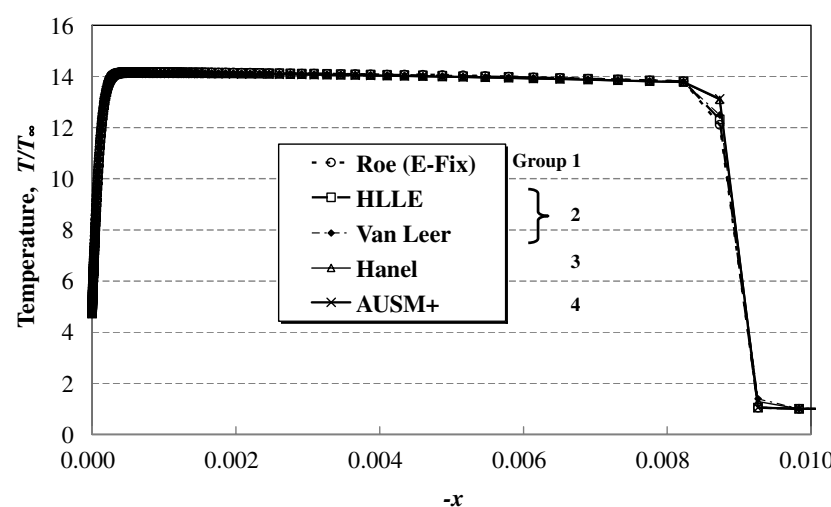

a)

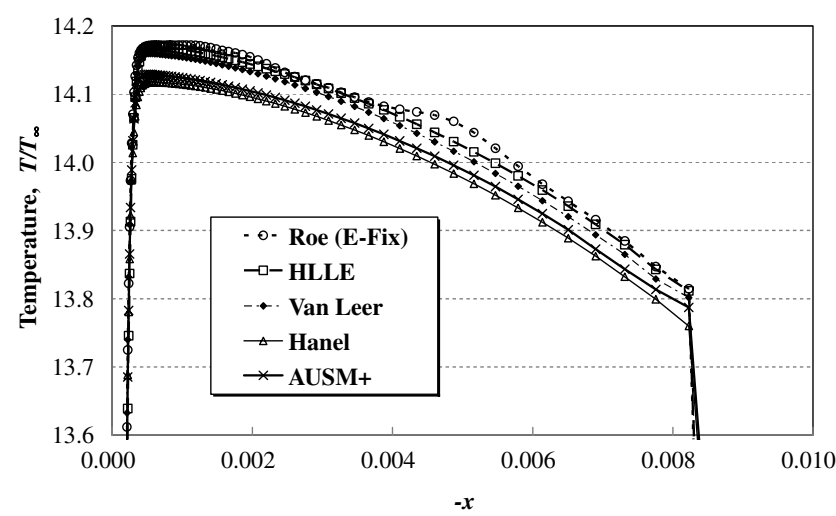

c)

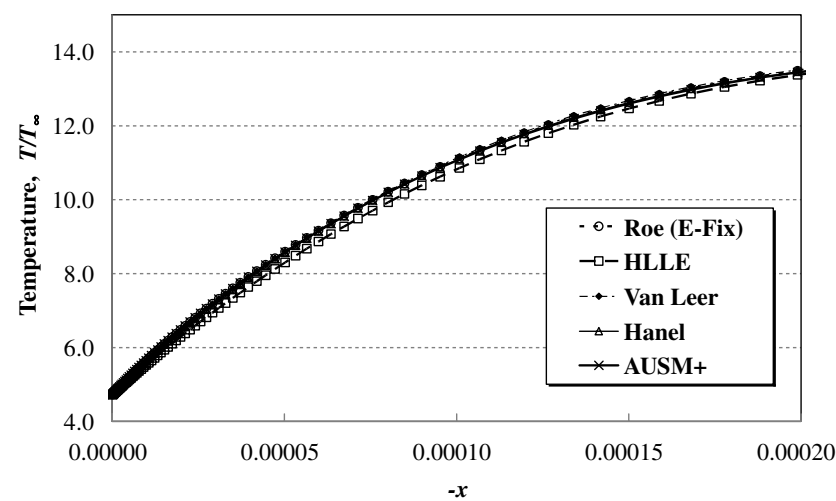

e)

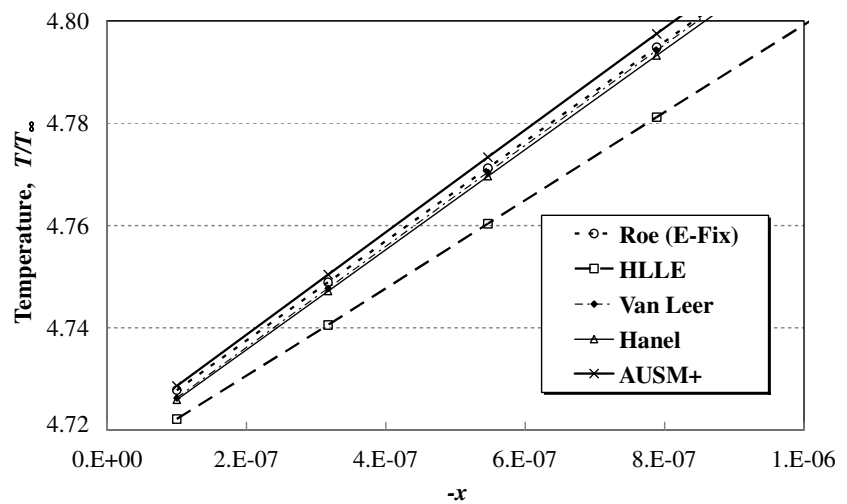

g)

Fig. 10 Temperature (left) and total enthalpy (right) profiles along symmetry line for blunt-body viscous test with different scales (100,000 steps, baseline grid).

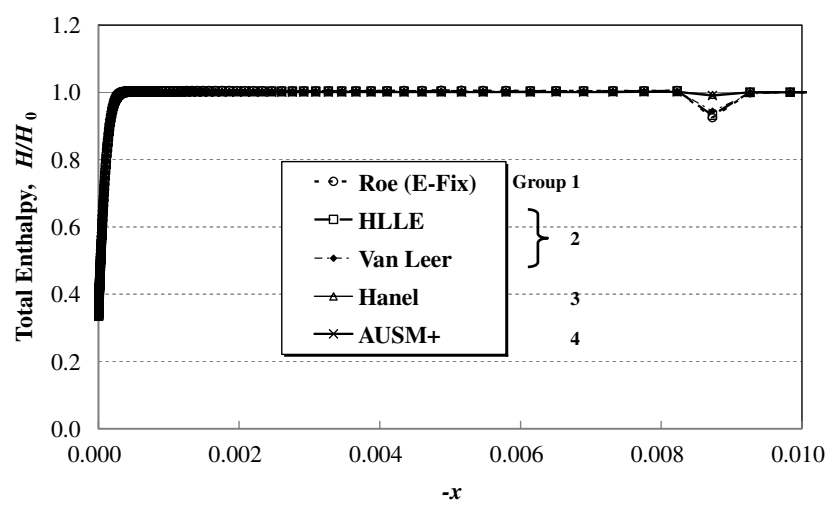

b)

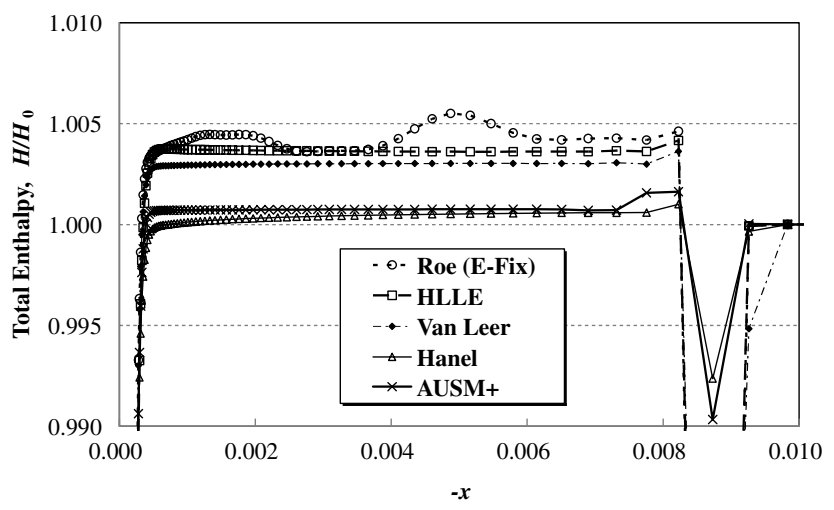

d)

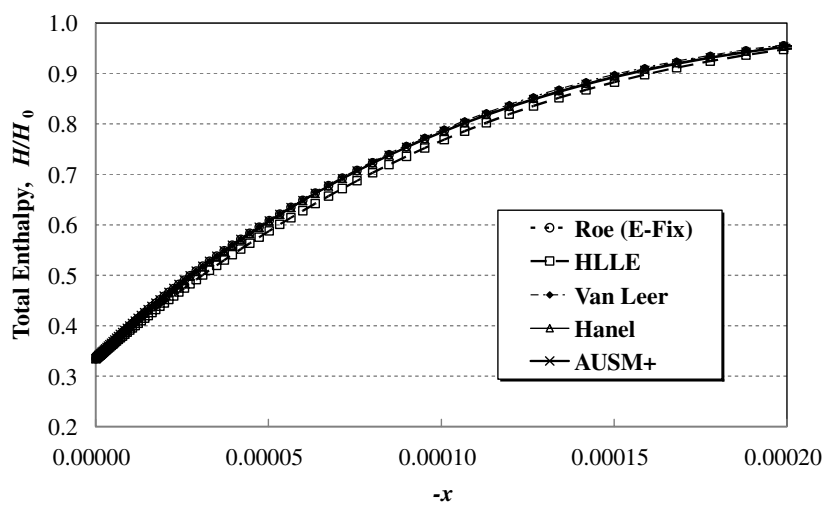

f)

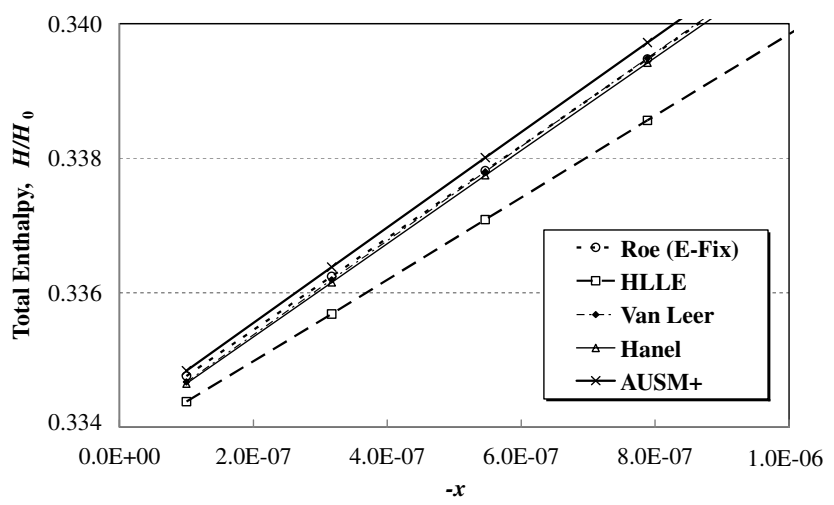

h) 
is taken as $R e_{\text {cell }}=1.3$ in the baseline grid, which satisfies Klopfer and Yee's criterion of $R e_{\text {cell }} \leq 3$ [41].

\section{A. Baseline Grid}

The grid employed here is shown in Fig. 8a, in which $160 \times 160$ cells are used. Shown in Fig. 8 b is the coordinate system whose origin is located at the cylinder stagnation point (nose), and the angle $\phi$ is taken as $-75 \mathrm{deg}<\phi<+75 \operatorname{deg}(\varphi=0 \mathrm{deg}$ corresponds to the nose).

The following flux functions are employed: Roe (e-fix) and ECRoe $(\alpha=0.8)$ for group 1, HLLE and van Leer [16] for group 2, Hänel [13] for group 3, and AUSM+, AUSMPW+, and RoeM for group 4. All the computations were conducted for 100,000 steps with $\mathrm{CFL}=200$ using LU-SGS. The residuals (L2-norm of density) dropped at least 3 orders of magnitude for most of the cases. For the Roe (e-fix), EC-Roe $(\alpha=0.8)$, and RoeM cases, the residuals stagnated around at the 2 orders of reduction from the initial stage. The solutions are shown in Figs. 8c-8f.

Roe (e-fix) and EC-Roe $(\alpha=\overline{0} \overline{8})$ yielded wiggles that were evidences of shock anomalies, and AUSM+ exhibited slight oscillations near the shock away from the symmetry line, which is often experienced by this flux [ 5$]$. RoeM showed slight asymmetry. The results of the other fluxes seemed satisfactory.

Figure 9 presents surface pressure and heat transfer rate profiles. The horizontal axis is the angle $\phi$ defined in Fig. 8b. All the fluxes except for Roe (e-fix) and EC-Roe $(\alpha=0.8)$ in group 1 gave identical pressure profiles, and the stagnation values agree well with theoretical ones (pitot pressure, $P_{10} / P_{\infty}=84.9$ ). For heat transfer, however, only AUSM+ and AUSMPW + (group 4) gave accurate stagnation value (Fay-Riddell [42]'s theoretical value, $q_{\mathrm{FR}}=$ $17.5 \mathrm{~W} / \mathrm{cm}^{2}$ ) along with smooth distributions. Results of HLLE, van Leer [16] (group 2), and Hänel [13] (group 3) are smooth but underpredicted. The other fluxes [Roe (e-fix), EC-Roe $(\alpha=0.8)$, and RoeM] showed poor distributions due to shock anomalies.

Shown in Fig. 10 are temperature and total enthalpy profiles for Roe (e-fix) in group 1, HLLE and van Leer in group 2, Hänel [13] in group 3 , and AUSM+ in group 4 on the $j=80$ cells $(y \approx 0)$. Note that the horizontal axis $(-x)$ is positive and stands for the distance from the origin (stagnation point) toward the incoming flow. It is seen from Figs. 10a and 10b that all four groups showed similar trends with small differences near the shock $(-x \approx 0.009)$. These differences are more clearly seen in blowup views of Figs. 10c and 10d, in which total enthalpies (Fig. 10d) past the shock deviated much more significantly (roughly one order larger magnitude) in non- $H$-preserving fluxes [Roe (e-fix), HLLE, and van Leer] than $H$ preserving fluxes (Hänel [13] and AUSM+), and this affected temperature profiles (Fig. 10c). In the boundary layer near the wall (Figs. 10e and 10f), however, the deviations in non- $H$-preserving fluxes seemed to recover, as expected from the discussions in Sec. III.B except for HLLE. In Figs. $10 \mathrm{~g}$ and $10 \mathrm{~h}$, only four cells near the wall are displayed for clarity, and all the results showed linear profiles. This means that enough grid resolution in the thermal boundary layer is achieved in the present setup. In Fig. $10 \mathrm{~g}$, AUSM+, which gave the most accurate surface heating (Fig. $\overline{\text { 9d) }}$, showed the steepest temperature gradient, followed by Roe (e-fix), Hänel [13], and finally, HLLE, which gave the lowest surface heating (Fig. 9c).

According to these results, the following conclusions can be drawn for the three properties:

1) Property 1, shock stability/robustness: Roe (e-fix) and EC-Roe ( $\alpha=0.8$ ) of group 1 both showed anomalous distributions of heating in Fig. 9c. This property is obviously crucial for heating computations.

2) Property 2, $\mathrm{H}$-preserving: comparing the results of van Leer [16] (group 2) and Hänel [13] (group 3), the $H$-preserving property

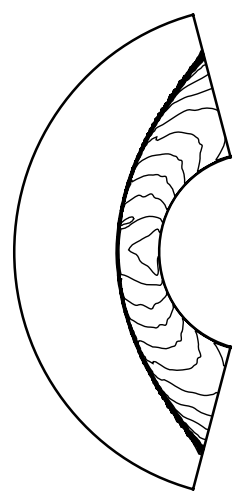

a)

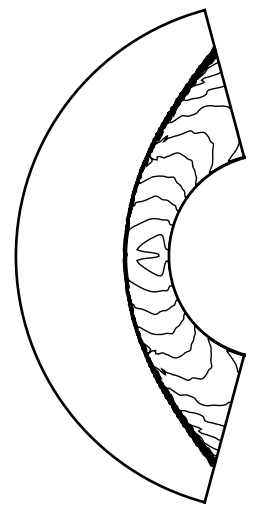

b)

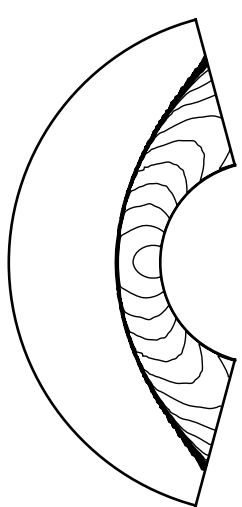

c)

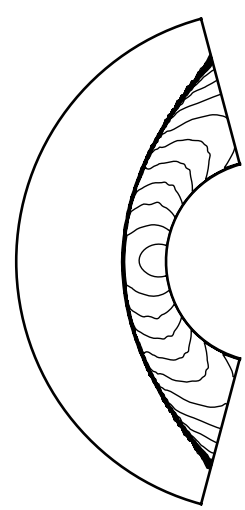

d)

Fig. 11 Mach number contours of a) Roe (e-fix), b) AUSM+, c) AUSMPW+, and d) RoeM for blunt-body viscous test (100,000 steps, fine grid).

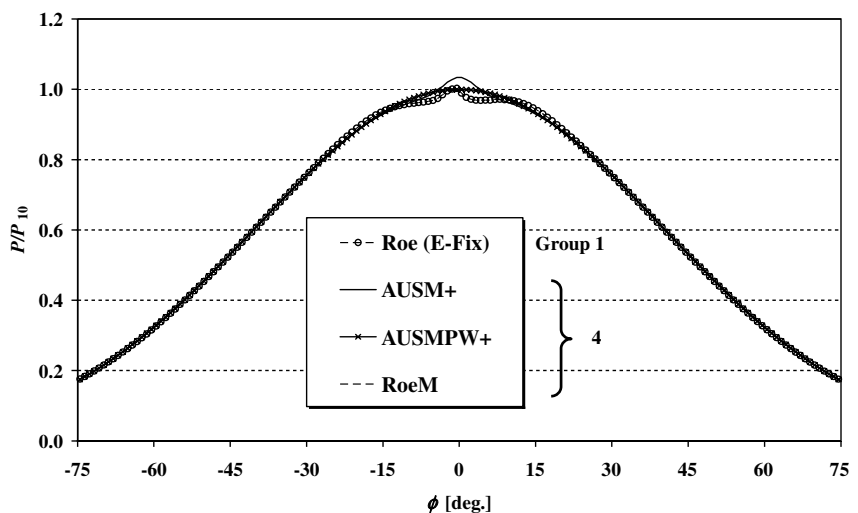

a)

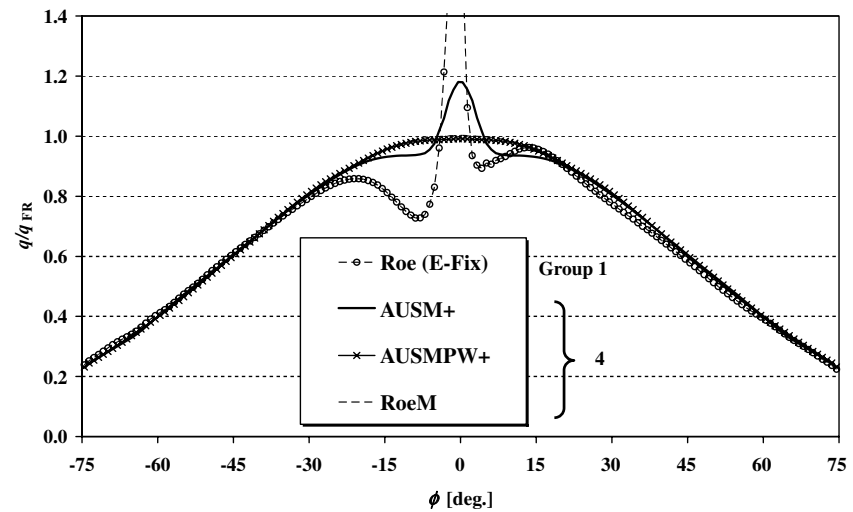

b)

Fig. 12 Profiles of a) pressure and b) heat transfer rates over blunt body $(100,000$ steps, fine grid). 


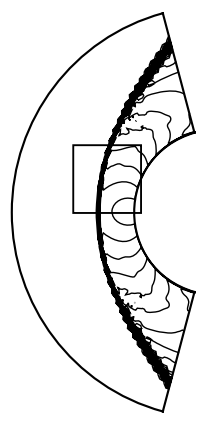

a)

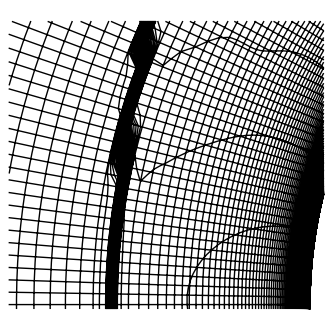

Fig. 13 Comparison of Mach number contours of AUSM+ results of a) baseline and b) fine grids for blunt-body viscous tests at 100,000 steps.

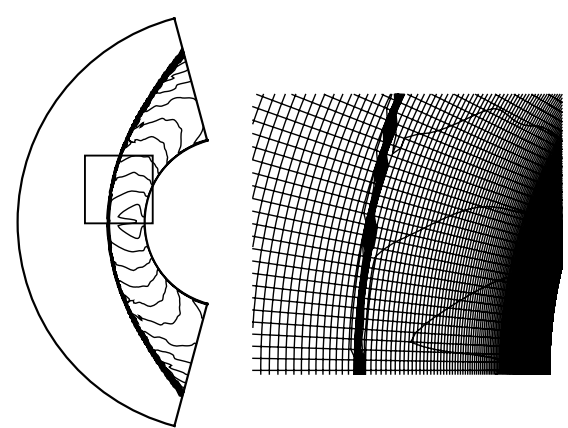

b) (property 2) of Hänel seems to have minor effects on the calculated surface heating. According to Fig. 10d, Hänel has less than $0.1 \%$ error of $H$, which showed slight improvement over van Leer (about $0.3 \%$ ). As a result, differences of the heating (Fig. 9c) and temperature gradient (Fig. 10g) are smaller than 2\%, which is not significant compared with other flux functions.

3) Property 3, boundary-layer resolution: this property also seems crucial according to the results of Hänel [13] (group 3) and AUSM+ (group 4). Although Hänel exhibited the least error of $H$ behind the shock (less than $0.1 \%$ in Fig. 10d), the resultant heating showed at most $17 \%$ error (Fig. 9c).

In summary, properties 1 and 3 are crucial, whereas property 2 is seemingly preferred to predict hypersonic surface heating accurately. This was reflected in the scaling of these properties in Tables 3 and 4 : one-dimensional and MD shock stabilities of property 1 have $\overline{20}$ points in maximum for each, 10 points for property 2 , and 30 points for property 3 . According to this table, group 4 fluxes are suitable for hypersonic heating computations.

\section{B. Fine Grid for Additional Discussions}

Finally, group 4 fluxes, along with Roe (e-fix) for reference, are applied for a finer grid in which the number of cells only in the direction normal to the wall ( $j$ direction) has been doubled $\left(320 \times 160\right.$ cells, $\left.R e_{\text {cell }} \approx 0.7\right)$. The computations were conducted for 100,000 steps with CFL $=400$. All the computations showed at least two orders of residual reductions.

The pressure contours and surface pressure/heating profiles are shown in Figs. 11 and 12, respectively. According to Fig. 11, Roe (e-fix) showed an anomalous result again. AUSM+, which showed a stable result at 50,000 steps (not shown), exhibited shock anomalies at 100,000 steps. This is consistent with the finding in [4], in which AUSM+ showed MD anomalies grown subliminally and very gently while the solution was apparently satisfactory. For AUSMPW + and RoeM, there is no evidence of shock anomalies. In Fig. 12, as can be expected, Roe (e-fix) and AUSM+ yielded small errors in pressure and significant errors in heat transfer rates. AUSMPW + and RoeM showed identical results both in pressure and heating and excellent agreement with theory at stagnation. It is noted that AUSM+, which showed a stable result on the baseline grid, exhibited anomalies on the fine grid; on the other hand, RoeM, which suffered from weak shock oscillations on the baseline grid, showed an improvement of the solution on the fine grid.

The failure of AUSM+ in this case is explained by the relative positioning of the captured shocks on the grid, following the discussion in [4]. Shown in Fig. 13 are blowup views of the AUSM+ results both on the baseline and the fine grids. Note that the captured shock is thicker in the baseline case than the fine grid case, and this seemed to provide the shock with sufficient amount of dissipation that successfully suppressed the oscillations in the shock-normal direction (one-dimensional stable). On the fine grid, however, the shock was more likely to move back and forth near the line of symmetry, and it jumped from one set of a grid line to another. This would be the cause of the oscillation mode in this case. This jump was also seen in the baseline case, but away from the symmetry line, so its effect on the solution was subtle. This discussion is what we mentioned earlier in Sec. III.A; that is, clustering the grids in the shock-normal direction may or may not result in worse solutions. From this viewpoint, it would be better to use a one-dimensional stable flux, such as Roe (e-fix) and EC-Roe $(\alpha=0.8)$, in combination with MD dissipation $[\underline{43}, \underline{44}]$, which can suppress the additional mode associated with, for example, the jumps of the shock between grid lines, though we have not completed to design it. AUSMPW + was the only method that showed stable results both on the two grids in the present cases, and this explains the highest score of this flux in Tables 4 . This is perhaps due to its MD dissipation term, but this flux may exhibit the shock anomalies on another grid, according to [4]. Nevertheless, it is confirmed that some of group 4 fluxes are more robust (i.e., property 1 is likely to be satisfied) than others, and have properties 2 and 3, so would be the most reliable methods for hypersonic heating computations at this stage. Roe (e-fix) and EC-Roe $(\alpha=0.8)$ in group 1 are also promising if an aforementioned MD dissipation is established for practical use [43]. Thus, we are currently engaged in developing a flux function of group 4 with MD dissipation $[\underline{45}, \underline{46}]$.

\section{Conclusions}

An extensive detailed evaluation of Euler fluxes has been conducted in the present study for hypersonic surface heating computations. We first proposed the following three properties for flux functions: 1) shock stability/robustness, 2) conservation of total enthalpy (and hence total temperature), and 3) resolving boundary layer (and hence temperature gradient).

It turned out that no flux functions investigated here possessed all three properties. In particular, the first one is not satisfied by any flux functions, including flux-vector-splittings. These fluxes were believed to be carbuncle free, but even they exhibit multidimensional shock anomalies for a mesh either with a large numbers of cells in the shock-parallel direction, or a large cell AR. It is confirmed that the second property is strongly satisfied by fluxes that were designed to do so, but the other fluxes also provided comparable results to those fluxes in second-order-accurate computations. However, the secondorder extension did not compensate the lack of the third property unless the flux was formulated to resolve contact discontinuities.

According to how these properties are satisfied, we categorized 15 popular or recently developed flux functions into the following five groups:

1) Group 1 (exact or three-wave approximate Riemann fluxes): Godunov, Roe, Roe (e-fix), and EC-Roe. Property 2 is no, and property 3 is yes.

2) Group 2 (two-wave approximate Riemann fluxes): HLLE and van Leer [16]. Property 2 is no, and property 3 is no.

3) Group 3 (total enthalpy-preserving, two-wave approximate Riemann flux): Hänel [13]. Property 2 is yes, and property 3 is no.

4) Group 4 (total enthalpy-preserving fluxes): AUSMDV, AUSM+, $\mathrm{AUSM}^{+}$-up, AUSMPW+, and RoeM. Property 2 is yes, and property 3 is yes.

5) Group 5 (hybrid fluxes): AUSMDV (shock fix) and rotatedRHLL. 
Then these fluxes are applied for two-dimensional, viscous, hypersonic flow computations over a blunt body. It is concluded from the results that the first and the third properties are crucial, and the second one is preferred to predict hypersonic surface heating accurately.

According to the present survey, group 4 fluxes such as AUSMPW + appeared to be promising for use, because these fluxes are relatively robust among the currently available fluxes (i.e., the first property is more likely to be satisfied than others) and possess the rest of the properties.

In designing a flux function, it is recommended to develop or improve a flux of group 4 or group 1 with multidimensional dissipation, which is readily applicable to unstructured grids [4346], or in combination with a dissipative flux in a multidimensional and systematic manner [30].

\section{Acknowledgments}

We are grateful to Hiroaki Nishikawa, National Institute of Aerospace, for reviewing the earlier version of the present paper, and giving us constructive comments. Meng-Sing Liou at NASA John $\mathrm{H}$. Glenn Research Center at Lewis Field provided us with valuable advice that led to significant improvement of the present article. Peter Gnoffo, NASA Langley Research Center, provided helpful information. We appreciate their cooperation. We also thank Taku Nonomura, University of Tokyo (currently at Japan Aerospace Exploration Agency), for having interesting discussions with us.

\section{References}

[1] Peery, K. M., and Imlay, S. T., "Blunt-Body Flow Simulations," AIAA Paper 88-2904, 1988.

[2] Pandolfi, M., and D'Ambrosio, D., "Numerical Instabilities in Upwind Methods: Analysis and Cures for the Carbuncle Phenomenon," Journal of Computational Physics, Vol. 166, No. 2, 2001, pp. 271-301. doi: $10.1006 / j \mathrm{jcph} .2000 .6652$

[3] Barth, T. J., "Some Notes on Shock-Resolving Flux Functions Part 1: Stationary Characteristics," NASA TM-101087, 1989.

[4] Kitamura, K., Roe, P., and Ismail, F., "Evaluation of Euler Fluxes for Hypersonic Flow Computations," AIAA Journal, Vol. 47, No. 1, 2009, pp. 44-53. doi:10.2514/1.33735

[5] Kim, S. S., Kim, C., Rho, O. H., and Hong, S. K., "Methods for the Accurate Computations of Hypersonic Flows I. AUSMPW+ Scheme," Journal of Computational Physics, Vol. 174, No. 1, 2001, pp. 38-80. doi:10.1006/jcph.2001.6873

[6] Kim, K. H., Kim, C., and Rho, O. H., "Cures for the Shock Instability, Development of a Shock-Stable Roe Scheme," Journal of Computational Physics, Vol. 185, No. 2, 2003, pp. 342-374. doi:10.1016/S0021-9991(02)00037-2

[7] Henderson, S. J., and Menart, J. A., "Grid Study on Blunt Bodies with the Carbuncle Phenomenon," AIAA Paper 2007-3904, 2007.

[8] Quirk, J. J., "A Contribution to the Great Riemann Solver Debate," International Journal for Numerical Methods in Fluids, Vol. 18, No. 6, 1994 , pp. $555-574$. doi:10.1002/fld.1650180603

[9] Gnoffo, P. A., and White, J. A., "Computational Aerothermodynamic Simulation Issues on Unstructured Grids," AIAA Paper 2004-2371, 2004.

[10] Mazaheri, A. R., and Kleb, B., "Exploring Hypersonic, UnstructuredGrid Issues Through Structured Grids," AIAA Paper 2007-4462, 2007.

[11] Roe, P. L., "Approximate Riemann Solvers, Parameter Vectors, and Difference Schemes," Journal of Computational Physics, Vol. 43, No. 2, 1981, pp. 357-372. doi:10.1016/0021-9991(81)90128-5

[12] Liou, M. S., "A Sequel to AUSM: AUSM+," Journal of Computational Physics, Vol. 129, No. 2, 1996, pp. 364-382. doi:10.1006/jcph.1996.0256

[13] Hänel, D., Schwane, R., and Seider, G., "On the Accuracy of Upwind Schemes for the Solution of the Navier-Stokes Equations," AIAA Paper 87-1105, 1987.

[14] Jameson, A., "Analysis and Design of Numerical Schemes for Gas Dynamics, 2: Artificial Diffusion and Discrete Shock Structure," International Journal of Computational Fluid Dynamics, Vol. 5, Nos. 1-2, 1995, pp. 1-38. doi: $10.1080 / 10618569508940734$
[15] Steger, J. L., and Warming, R. F., "Flux Vector Splitting of the Inviscid Gasdynamics Equations with Application to Finite Difference Methods," Journal of Computational Physics, Vol. 40, No. 2, 1981, pp. 263-293. doi:10.1016/0021-9991(81)90210-2

[16] van Leer, B., "Flux Vector Splitting for the Euler Equations," Lecture Notes in Physics, Vol. 170, No., 1982, pp. 507-512. doi:10.1007/3-540-11948-5_66

[17] Roe, P. L., Nishikawa, H., Ismail, F., and Scalabrin, L., "On Carbuncles and Other Excrescences," AIAA Paper 2005-4872, 2005.

[18] van Leer, B., "Towards the Ultimate Conservative Difference Scheme 5: A Second-Order Sequel to Godunov's Method," Journal of Computational Physics, Vol. 32, No. 1, 1979, pp. 101-136. doi:10.1016/0021-9991(79)90145-1

[19] van Albada, G. D., van Leer, B., and Roberts, W. W., Jr., "AComparative Study of Computational Methods in Cosmic Gas Dynamics," Astronomy and Astrophysics, Vol. 108, No. 1, 1982, pp. 76-84.

[20] Godunov, S. K., "A Finite Difference Method for the Numerical Computation of Discontinuous Solutions of the Equations of Fluid Dynamics," Matematicheskii Sbornik / Izdavaemyi Moskovskim Matematicheskim Obshchestvom, Vol. 47, No. 3, 1959, pp. 271-306.

[21] Harten, A., "High Resolution Schemes for Hyperbolic Conservation Laws," Journal of Computational Physics, Vol. 49, No. 3, 1983, pp. 357-393. doi:10.1016/0021-9991(83)90136-5

[22] Ismail, F., "Toward a Reliable Prediction of Shocks in Hypersonic Flow -Resolving Carbuncles with Entropy and Vorticity Control," Ph.D. Dissertation, Univ. of Michigan, Ann Arbor, Michigan, 2006.

[23] Ismail, F., Roe, P. L., and Nishikawa, H., "A Proposed Cure to the Carbuncle Phenomenon," U18: The Fourth International Conference on Computational Fluid Dynamics, , Ghent, Belgium, 2006.

[24] Roe, P. L., "Affordable, Entropy-Consistent Euler Flux Functions 1: Analytical Results," Journal of Computational Physics (submitted for publication).

[25] Ismail, F., and Roe, P. L., "Affordable, Entropy-Consistent Euler Flux Functions 2: Entropy Production at Shocks," Journal of Computational Physics, Vol. 228, No. 15, 2009, pp. 5410-5436. doi:10.1016/j.jcp.2009.04.021

[26] Einfeldt, B., "On Godunov-Type Methods for Gas Dynamics," SIAM Journal on Numerical Analysis, Vol. 25, No. 2, 1988, pp. 294-318. doi:10.1137/0725021

[27] Wada, Y., and Liou, M. S., "An Accurate and Robust Flux Splitting Scheme for Shock and Contact Discontinuities," SIAM Journal on Scientific Computing, Vol. 18, No. 3, 1997, pp. 633-657. doi:10.1137/S1064827595287626

[28] Liou, M. S., and Steffen, C. J., Jr., "A New Flux Splitting Scheme," Journal of Computational Physics, Vol. 107, No. 1, 1993, pp. 23-39. doi:10.1006/jcph.1993.1122

[29] Liou, M. S., "A Further Development of the AUSM+ Scheme Towards Robust and Accurate Solutions for All Speeds," AIAA Paper 20034116, 2003.

[30] Nishikawa, H., and Kitamura, K., "Very Simple, Carbuncle-Free, Boundary-Layer-Resolving, Rotated-Hybrid Riemann Solvers," Journal of Computational Physics, Vol. 227, No. 4, 2008, pp. 2560 2581. doi:10.1016/j.jcp.2007.11.003

[31] Yoon, S., and Jameson, A., "Lower-Upper Symmetric-Gauss-Seidel Method for the Euler and Navier-Stokes Equations," AIAA Journal, Vol. 26, No. 9, 1988, pp. 1025-1026.

[32] Roe, P. L., "Fluctuations and Signals-A Framework for Numerical Evolution Problems," Numerical Methods for Fluid Dynamics, edited by K. W. Morton, and M. J. Baines, Academic Press, New York, 1982, pp. 232-236.

[33] Hirsch, C., Numerical Computation of Internal and External Flows, Volume 2: Computational Methods for Inviscid and Viscous Flows, Wiley, New York, 1990, p. 424.

[34] Gnoffo, P., Buck, G., Moss, J., Nielsen, E., Berger, K., and Jones, W. T., "Aerothermodynamic Analyses of Towed Ballutes," AIAA Paper 20063771, 2006.

[35] Yamamoto, Y., Nagai, S., Koyama, T., Tsuda, S., Hirabayashi, N., and Hozumi, K., "CFD Analysis and Wind Tunnel Experiments of Hypersonic Shock-Shock Interaction Heating for Two Hemisphere Cylinder Problem," AIAA Paper 2002-0217, 2002.

[36] Houwing, A. F. P., Smith, D. R., Fox, J. S., Danehy, P. M., and Mudford, N. R., "Laminar Boundary Layer Separation at a Fin-Body Junction in a Hypersonic Flow," Shock Waves, Vol. 11, No. 1, 2001, pp. 31-42. doi:10.1007/PL00004055

[37] Edney, B., "Anomalous Heat Transfer and Pressure Distributions on Blunt Bodies at Hypersonic Speed in the Presence of an Impinging 
Shock," Aeronautical Research Inst., Federal Aviation Administration, Rept. 115, Sweden, 1968.

[38] Wieting, A. R., and Holden, M. S., "Experimental Shock-Wave Interference Heating on a Cylinder at Mach 6 and 8," AIAA Journal, Vol. 27, No. 11, 1989, pp. 1557-1565. doi: $10.2514 / 3.10301$

[39] Kitamura, K., Shima, E., and Roe, P. L., "Three-Dimensional Carbuncles and Euler Fluxes," AIAA Paper 2010-1273, 2010.

[40] Nishino, A., Ishikawa, T., Kitamura, K., and Nakamura, Y., "Effect of the Clearance Between Two Bodies on TSTO Aerodynamic Heating," Journal of Japan Society for Aeronautical and Space Sciences, Vol. 53, No. 622, 2005, pp. 503-509 (in Japanese).

[41] Klopfer, G. H., and Yee, H. C., "Viscous Hypersonic Shock-on-Shock Interaction on Blunt Cowl Lips," AIAA Paper 88-0233, 1988.

[42] Fay, J. A., and Riddell, F. R., "Theory of Stagnation Point Heat Transfer in Dissociated Air," Journal of the Aeronautical Sciences, Vol. 25,
No. 2, 1958, pp. 73-85.

[43] Roe, P. L., and Kitamura, K., "Artificial Surface Tension to Stabilize Captured Shockwaves," AIAA Paper 2008-3991, 2008.

[44] Gnoffo, P. A., "Multi-Dimensional, Inviscid Flux Reconstruction for Simulation of Hypersonic Heating on Tetrahedral Grids," AIAA Paper 2009-599, 2009.

[45] Shima, E., and Kitamura, K., "On New Simple Low-Dissipation Scheme of AUSM-Family for All Speeds," AIAA Paper 2009-136, 2009.

[46] Shima, E., and Kitamura, K., "On AUSM-Family Scheme for All Speeds with Shock Detection for Carbuncle-Fix," AIAA Paper 2009$3544,2009$. 